\title{
Search at the CERN LHC for a light neutralino of cosmological interest *
}

\author{
S. Choi,${ }^{1}$ S. Scopel, ${ }^{2}$ N. Fornengo, ${ }^{3}$ and A. Bottino ${ }^{3}$ \\ ${ }^{1}$ Korea University, Seoul, Korea, 136-701 \\ ${ }^{2}$ Department of Physics, Sogang University \\ Seoul, Korea, 121-742 \\ ${ }^{3}$ Dipartimento di Fisica Teorica, Università di Torino \\ Istituto Nazionale di Fisica Nucleare, Sezione di Torino \\ via P. Giuria 1, I-10125 Torino, Italy
}

(Dated: May 11, 2018)

\begin{abstract}
We address the problem of a search at the LHC for a neutralino whose mass is around $10 \mathrm{GeV}$, i.e. in the range of interest for present data of direct search for dark matter particles in the galactic halo. This light neutralino is here implemented in an effective Minimal Supersymmetric extension of the Standard Model at the electroweak scale without requirement of a gaugino-mass unification at a grand unification scale. Within this model we select a representative benchmark and determine its prospects of reconstructing the main features of the model at different stages of the LHC runs.

PACS numbers: 95.35.+d,11.30.Pb,12.60.Jv,95.30.Cq
\end{abstract}

\section{INTRODUCTION}

Most investigations on the search for neutralinos at the CERN Large Hadron Collider (LHC) concern neutralinos within Supergravity-inspired (SUGRA) models. This implies, in particular, neutralinos of a mass larger than about $50 \mathrm{GeV}$, since this is the lower bound on the neutralino mass which directly follows from the LEP lower bound on the chargino mass combined with the assumption, inherent in SUGRA models, that the gaugino masses are unified at a grand unification (GUT) scale.

Relaxation of this hypothesis of gaugino-mass unification allows the neutralino mass $m_{\chi}$ to be smaller than $50 \mathrm{GeV}$. A supersymmetric model which incorporates this possibility and is still very manageable, since expressible in terms of a limited number of independent parameter, is the one considered in Ref. [1]. It consists of an effective Minimal Supersymmetric extension of the Standard Model (MSSM) at the electroweak (EW) scale, whose main properties are summarized in Sect. [1]

In Ref. [1] it was stressed that, in case of R-parity conservation, a light neutralino (i.e. a neutralino with $m_{\chi} \lesssim 50$ $\mathrm{GeV}$ ), when happens to be the Lightest Supersymmetric Particle (LSP), constitutes an extremely interesting candidate for the dark matter (DM) in the Universe, with direct detection rates accessible to experiments of present generation.

In Ref. [1] it was also derived a lower bound for $m_{\chi}$ from the cosmological upper limit on the cold dark matter density; the value of this lower bound, updated on the basis of the experimental data available in Autumn 2010, was established in Ref. [2] to be $m_{\chi} \sim 7.5 \mathrm{GeV}$. The implementation of the very recent upper bound on the branching ratio for the process $B R\left(B_{s}^{0} \rightarrow \mu^{-}+\mu^{+}\right)[3]$ moves now the lower bound on the neutralino mass to the value $m_{\chi} \sim 9$ $\mathrm{GeV}$. The theoretical framework which allows neutralinos with a mass in the range $9 \mathrm{GeV} \lesssim m_{\chi} \lesssim 50 \mathrm{GeV}$ is briefly summarized in Sect. II and will be simply denominated as Light Neutralino Model (LNM) in the present paper.

In Ref. [4] the LNM was proved to fit the annual modulation effect measured by the DAMA collaboration [5]. Our model is also compatible with all experimental searches for indirect evidence of SUSY and with precision data that set constraints on possible effects due to supersymmetry, as discussed in detail in Ref. 2] (for the compatibility of very light neutralino masses with various laboratory bounds see also Ref. [6]). Moreover, the possible impact of some early analyses by the CMS and ATLAS Collaborations at the LHC on the LNM was investigated in Ref. 7].

At the same time much interest has recently been raised by a new measurement of an annual modulation effect by the CoGeNT Collaboration [8] and by some hints of possible signals of dark matter (DM) particles in other experiments of direct detection (CDMS [9], CRESST [10]).

*Preprint number: DFTT 21/2011 
What is intriguing in all the experimental results listed above is that, if actually due to a Weakly Interacting Massive Particle (WIMP) with a coherent interaction with the atomic nuclei of the detector material, they would all be explained by a WIMP physical region with a light mass (around $10 \mathrm{GeV}$ ) and a nucleon elastic cross-section in agreement with the intervals for the same parameters established by the DAMA Collaboration from a measurement of the annual modulation that has now reached a high statistical significance by a running over 13 yearly cycles with the DAMA/NaI and the DAMA/LIBRA experiments [11]. Specifically, compatibility of the DAMA result with CDMS has been discussed in Ref. [12], and between CoGeNT and DAMA in Ref. [13].

These results have prompted a large number of phenomenological papers focused on WIMPs with a light mass [14]. Turning to a specific candidate, it has now become common to consider neutralinos with a mass of order $10 \mathrm{GeV}$.

Due to the relevant role that light neutralinos can have in cosmology and astrophysics it becomes so of the upmost interest to investigate the possibility of searching for these particles at the LHC. A preliminary analysis in this direction was performed in Ref. [15]; event rates were determined in specific scenarios and benchmarks dictated by the relevant cosmological properties of the LNM. There it was shown that the perspectives of a fruitful investigation of the supersymmetric parameter space relevant for light neutralinos at the LHC are potentially good, though no specific analyses of the signal/background ratios and of kinematical distributions were performed.

In the present paper we wish to extend the investigation of Ref. 15] by making use of a numerical simulation to estimate in a realistic way the detectability of the LNM at the LHC over the SM background and to show what information about the masses of SUSY particles can be extracted from the data.

The paper is organized as follows. In Section [I] we summarize the main features of the LNM, concentrating on the particle mass spectra relevant for our analysis. In Section [III we discuss the general properties in the LNM of the decay chains that are used to reconstruct the SUSY masses. In Section IV we explore the LNM parameter space to select a suitable benchmark for our scenario, that is then used in Section $\nabla$ to assess its chances for an early discovery within the $7 \mathrm{TeV}$ run at the LHC, and in Section VI to discuss how the SUSY masses can be reconstructed from invariant-mass spectra measured with a larger collected luminosity at $14 \mathrm{TeV}$. We then give our conclusions in Section VII]

\section{A MODEL FOR LIGHT NEUTRALINOS (LNM)}

The supersymmetric scheme we employ in the present paper is an effective MSSM scheme (effMSSM) at the electroweak scale, with the following independent parameters: $M_{1}, M_{2}, M_{3}, \mu, \tan \beta, m_{A}, m_{\tilde{q}}, m_{\tilde{t}}, m_{\tilde{l}}$ and $A$. Notations are as follows: $M_{1}, M_{2}$ and $M_{3}$ are the $\mathrm{U}(1), \mathrm{SU}(2)$ and $\mathrm{SU}(3)$ gaugino masses (these parameters are taken here to be positive), $\mu$ is the Higgs mixing mass parameter, $\tan \beta$ the ratio of the two Higgs v.e.v.'s, $m_{A}$ the mass of the CP-odd neutral Higgs boson, $m_{\tilde{q}}$ is a squark soft-mass common to the squarks of the first two families, $m_{\tilde{t}}$ is the squark soft-mass of the third family, $m_{\tilde{l}}$ is a slepton soft-mass common to all sleptons, and $A$ is a common dimensionless trilinear parameter for the third family, $A_{\tilde{b}}=A_{\tilde{t}} \equiv A m_{\tilde{t}}$ and $A_{\tilde{\tau}} \equiv A m_{\tilde{l}}$ (the trilinear parameters for the other families being set equal to zero). In our model, no gaugino mass unification at a Grand Unified (GUT) scale is assumed: this implies that $M_{1}$ and $M_{2}$ are independent parameters at the EW scale. The model introduced here is the one discussed in Ref. [1], with the minimal extension that the degeneracy between the soft squark mass of the first two families and that of the third family is removed. In particular, the splitting between $m_{\tilde{q}}$ and $m_{\tilde{t}}$ reduces some tuning introduced in the parameter space by the constraint on the $b \rightarrow s+\gamma$ when $m_{\tilde{t}}=m_{\tilde{q}} \gtrsim 700 \mathrm{GeV}[7]$.

The following experimental constraints are imposed: accelerators data on supersymmetric and Higgs boson searches (CERN $e^{+} e^{-}$collider LEP2 [16], Collider Detectors D0 and CDF at Fermilab [17]); early bounds from Higgs searches at the LHC [18, 19]; measurements of the $b \rightarrow s+\gamma$ decay process [20]: $2.89 \leq B(b \rightarrow s+\gamma) \cdot 10^{4} \leq 4.21$ is employed here (this interval is larger by $25 \%$ with respect to the experimental determination [20] in order to take into account theoretical uncertainties in the supersymmetric (SUSY) contributions 21] to the branching ratio of the process (for the SM calculation, we employ the recent NNLO results from Ref. [22])); the measurements of the muon anomalous magnetic moment $a_{\mu} \equiv\left(g_{\mu}-2\right) / 2$ : for the deviation, $\Delta a_{\mu} \equiv a_{\mu}^{\exp }-a_{\mu}^{\text {the }}$, of the experimental world average from the theoretical evaluation within the SM we use here the $(2 \sigma)$ range $31 \leq \Delta a_{\mu} \cdot 10^{11} \leq 479$, derived from the latest experimental 23] and theoretical [24] data (the supersymmetric contributions to the muon anomalous magnetic moment within the MSSM are evaluated here by using the formulae in Ref. 25]); the upper bound on the branching ratio $B R\left(B_{s}^{0} \rightarrow \mu^{-}+\mu^{+}\right)$[3, 26]: we take $B R\left(B_{s}^{0} \rightarrow \mu^{-}+\mu^{+}\right)<1.5 \cdot 10^{-8}$; the constraints related to $\Delta M_{B, s} \equiv$ $M_{B_{s}}-M_{\bar{B}_{s}}[27$, 28]; the measurements of the decays $B \rightarrow \tau \nu$ [29] and $R(D) \equiv B R(B \rightarrow D \tau \nu) / B R(B \rightarrow D e \nu)$ [30] 
(in particular, the compatibility of very light neutralino masses with the latter four constraints is discussed in detail in Ref. [2]).

The linear superpositions of bino $\tilde{B}$, wino $\tilde{W}^{(3)}$ and of the two Higgsino states $\tilde{H}_{1}^{\circ}$, $\tilde{H}_{2}^{\circ}$ which define the four neutralino states, $\chi_{i}(\mathrm{i}=1,2,3,4)$ are written here as:

$$
\chi_{i} \equiv a_{1}^{(i)} \tilde{B}+a_{2}^{(i)} \tilde{W}^{(3)}+a_{3}^{(i)} \tilde{H}_{1}^{\circ}+a_{4}^{(i)} \tilde{H}_{2}^{\circ}
$$

The properties of these states have been investigated in detail, analytically and numerically, in Ref. [15] for the case when the smallest mass eigenstate $\chi_{1}$ (or $\chi$ in short) is light, i.e. $m_{\chi} \equiv m_{\chi_{1}} \lesssim 50 \mathrm{GeV}$. Of that analysis we report here only the main points which are relevant for the present paper.

We first notice that the lowest value for $m_{\chi}$ occurs when:

$$
m_{\chi} \simeq M_{1}<<|\mu|, M_{2}
$$

since the LEP lower limit on the chargino mass $\left(m_{\chi^{ \pm}} \gtrsim 100 \mathrm{GeV}\right)$ sets a lower bound on both $|\mu|$ and $M_{2}:|\mu|, M_{2} \gtrsim$ $100 \mathrm{GeV}$, whereas $M_{1}$ is unbound. Thus, $\chi \equiv \chi_{1}$ is mainly a Bino, whose mixings with the other interaction eigenstates are given by:

$$
\begin{aligned}
& \frac{a_{2}^{(1)}}{a_{1}^{(1)}} \simeq \frac{\xi_{1}}{M_{2}} \cot _{\theta}, \\
& \frac{a_{3}^{(1)}}{a_{1}^{(1)}} \simeq s_{\theta} s_{\beta} \frac{m_{Z}}{\mu} \\
& \frac{a_{3}^{(1)}}{a_{4}^{(1)}} \simeq-\frac{\mu s_{\beta}}{M_{1} s_{\beta}+\mu c_{\beta}},
\end{aligned}
$$

where $\xi_{1} \equiv m_{1}-M_{1}$. These expressions readily follow from the general analytical formulae given in Ref. [15] by taking $\tan \beta \geq 10$, as consistent with the scenarios discussed below.

Useful approximate expressions obtain also for the compositions of the eigenstates corresponding to the asymptotic mass eigenvalues: $m_{i} \sim \pm \mu$ and $m_{i} \sim M_{2}$. That is:

a) for the neutralino states $\chi_{i}$ with $m_{i} \simeq \pm \mu$,

$$
\begin{aligned}
& \frac{a_{2}^{(i)}}{a_{1}^{(i)}} \simeq \frac{ \pm \mu}{M_{2} \mp \mu} \cot _{\theta}, \\
& \frac{a_{1}^{(i)}}{a_{3}^{(i)}} \simeq \frac{2 \xi_{2} s_{\theta}\left( \pm \mu-M_{2}\right)}{M_{Z} s_{\beta}\left(s_{\theta}^{2} M_{2} \mp \mu\right)}, \\
& \frac{a_{3}^{(i)}}{a_{4}^{(i)}} \simeq \mp 1+\frac{\xi_{2}}{\mu},
\end{aligned}
$$

where $\xi_{2} \equiv \pm \mu-m_{i}$

b) for the neutralino state $\chi_{i}$ with $m_{i} \simeq M_{2}$,

$$
\begin{aligned}
& \frac{a_{1}^{(i)}}{a_{2}^{(i)}} \simeq \frac{\xi_{3}}{M_{2}} \tan _{\theta} \\
& \frac{a_{1}^{(i)}}{a_{3}^{(i)}} \simeq \frac{\xi_{3} s_{\theta}\left(M_{2}^{2}-\mu^{2}\right)}{M_{Z}\left(M_{2} c_{\beta}+\mu s_{\beta}\right) c_{\theta}^{2} M_{2}} \\
& \frac{a_{3}^{(i)}}{a_{4}^{(i)}} \simeq-\frac{\mu s_{\beta}+M_{2} c_{\beta}}{M_{2} s_{\beta}+\mu c_{\beta}}
\end{aligned}
$$


where $\xi_{3} \equiv M_{2}-m_{i}$.

From the above expressions the following relevant properties hold: (i) $\chi_{1}$ is mainly a B-ino whose mixing with $\tilde{H}_{1}^{\circ}$ is sizable at small $\mu$, (ii) $\chi_{3}$ has a mass $\left|m_{3}\right| \simeq|\mu|$ with a large $\tilde{H}_{1}^{\circ}-\tilde{H}_{2}^{\circ}$ mixing, independently of $M_{2}$, (iii) $\chi_{2}$ and $\chi_{4}$ interchange their main structures depending on the value of the ratio $|\mu| / M_{2}: \chi_{2}$ is dominantly a $\mathrm{W}$-ino (with a sizable subdominance of $\tilde{H}_{1}^{\circ}$ ) for $M_{2}<<|\mu|$ and a maximal $\tilde{H}_{1}^{\circ}-\tilde{H}_{2}^{\circ}$ admixture for $M_{2}>>|\mu|$, whereas $\chi_{4}$ is a maximal $\tilde{H}_{1}^{\circ}-\tilde{H}_{2}^{\circ}$ admixture for $M_{2}<<|\mu|$ and a very pure W-ino for $M_{2}>>|\mu|$.

For specific spectroscopic schemes, characterized by various internal hierarchies, we will use the denominations already introduced in Ref. [15], i.e.: (i) normal hierarchical scheme when $M_{2}<|\mu|$, (ii) degenerate scheme when $M_{2} \sim|\mu|$, (iii) inverted hierarchical scheme when $M_{2}>|\mu|$ (notice that we always assume $\left(M_{1}<<M_{2},|\mu|\right)$.

\section{A. Cosmologically inspired scenarios}

If light neutralinos are present in the Universe as relic particles, their abundance $\Omega_{\chi} h^{2}$ has to be smaller than the observed upper bound for cold dark matter $(\mathrm{CDM})$, i.e. $\Omega_{\chi} h^{2} \leq\left(\Omega_{C D M} h^{2}\right)_{\max }=0.122$ (this numerical value represents the $2 \sigma$ upper bound to $\left(\Omega_{C D M} h^{2}\right)_{\max }$ derived from the results of Ref. [31] ).

This requirement implies a lower limit on the neutralino pair annihilation cross section $\sigma_{\text {ann }}$ through the usual expression:

$$
\Omega_{\chi} h^{2}=\frac{x_{f}}{g_{\star}\left(x_{f}\right)^{1 / 2}} \frac{3.3 \cdot 10^{-38} \mathrm{~cm}^{2}}{<\widetilde{\sigma_{\text {ann }}} v>}
$$

where $\left\langle\widetilde{\sigma_{\text {ann }} v}\right\rangle \equiv x_{f}\left\langle\sigma_{\text {ann }} v\right\rangle_{\text {int }},\left\langle\sigma_{\text {ann }} v\right\rangle_{\text {int }}$ being the integral from the present temperature up to the freeze-out temperature $T_{f}$ of the thermally averaged product of the annihilation cross-section times the relative velocity of a pair of neutralinos, $x_{f}$ is defined as $x_{f} \equiv m_{\chi} / T_{f}$ and $g_{\star}\left(x_{f}\right)$ denotes the relativistic degrees of freedom of the thermodynamic bath at $x_{f}$.

The lower bound on $\sigma_{\text {ann }}$, implied by the cosmological upper limit on CDM, combined with the constraints due to accelerator data and other precision measurements, restricts markedly the overall size of the supersymmetric parameter space, as depicted in Figs. 1-2 of the second paper of Ref. [1]. In particular, it is instrumental in placing the fore mentioned limit $m_{\chi} \sim 9 \mathrm{GeV}$.

We thus arrive at the formulation of two specific physical scenarios for the case of light neutralinos of cosmological interest, as delineated in Ref. [15]. These scenarios are determined by the different ranges of the mass $m_{A}$ of the CP-odd neutral Higgs boson, and are summarized in Table \.

We have a Scenario $\mathcal{A}$ when $90 \mathrm{GeV} \leq m_{A} \lesssim(200-300) \mathrm{GeV}$ (we recall that $m_{A} \geq 90 \mathrm{GeV}$ is the LEP lower bound). When $m_{A}$ is in this range, then the neutralino mass can be as small as $\sim 9 \mathrm{GeV}$, since the cosmological upper bound is satisfied due to a sizable contribution to the neutralino pair annihilation cross section by the exchange of the A Higgs boson in the s channel. For this to be so, the B-ino component of the $\chi_{1}$ configuration must be maximally mixed with the $\tilde{H}_{1}^{\circ}$ component $\left(i\right.$. e. $\left.a_{3}^{(1)} / a_{1}^{(1)} \simeq 0.4\right)$. From the second expression in Eq. (4) one sees that this condition is satisfied when $\mu$ is small $(|\mu| \sim 100-200 \mathrm{GeV})$. Moreover, it turns out that $\tan \beta$ must be large $(\tan \beta \sim 30-45)$. The trilinear coupling is only mildly constrained to stay in the interval $-1 \lesssim A \lesssim+1$; the slepton soft mass $m_{\tilde{l}}$ and the squark soft mass $m_{\tilde{q}}$ are unconstrained. In this scenario, the following hierarchy holds for the coefficients $a_{i}^{(1)}$ of $\chi_{1}$ :

$$
\left|a_{1}^{(1)}\right|>\left|a_{3}^{(1)}\right|>>\left|a_{2}^{(1)}\right|,\left|a_{4}^{(1)}\right|
$$

as easily derivable from Eqs. (4).

When $m_{A} \gtrsim(200-300) \mathrm{GeV}$, the cosmological lower bound on $\sigma_{\text {ann }}$ can be satisfied by a pair annihilation process which proceeds through an efficient stau-exchange contribution (in the $t, u$ channels). This requires that: (i) the stau mass $m_{\tilde{\tau}}$ is sufficiently light, $m_{\tilde{\tau}} \sim 90 \mathrm{GeV}$ (notice that the current experimental limit is $m_{\tilde{\tau}} \sim 87 \mathrm{GeV}$ ) and (ii) $\chi_{1}$ is a very pure B-ino $\left(\right.$ i.e. $\left(1-a_{1}^{(1)}\right)=\mathrm{O}\left(10^{-3}\right)$. If this is the case, then light neutralinos can exist, but with a mass above $\sim 15-18 \mathrm{GeV}$ [1, 32]. As discussed in Ref. [15], conditions (i) and (ii) require that $|\mu| \gtrsim 500 \mathrm{GeV}, \tan \beta \lesssim$ $20 ; m_{\tilde{l}} \gtrsim(100-200) \mathrm{GeV}$; the parameter $A$ is typically in the range $-2.5 \lesssim A \lesssim+2.5$, the other supersymmetric 


\begin{tabular}{|c|c|c|c|c|c|}
\hline scenario & $M_{1}[\mathrm{GeV}]$ & $|\mu|[\mathrm{GeV}]$ & $\tan \beta$ & $m_{A}[\mathrm{GeV}]$ & $m_{\tilde{l}}[\mathrm{GeV}]$ \\
\hline \hline $\mathcal{A}$ & $\sim 10-14$ & $110-140$ & $30-45$ & $\sim 90-110$ & - \\
$\mathcal{B}$ & $\sim 25$ & $\gtrsim 500$ & $\lesssim 20$ & $\gtrsim 200$ & $100-200$ \\
\hline
\end{tabular}

TABLE I: Representative features for scenarios $\mathcal{A}$ and $\mathcal{B}$ described in Section IA In scenario $\mathcal{A}$ : $-1 \lesssim A \lesssim+1$, in scenario $\mathcal{B}$ : $-2 \lesssim A \lesssim+2$.
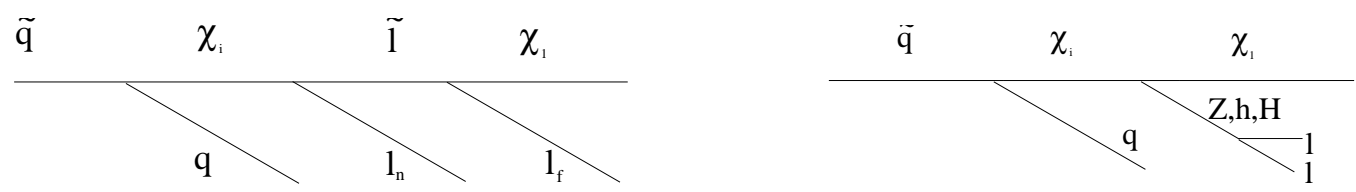

FIG. 1: Topologies of the decay $\tilde{q} \rightarrow q \chi \overline{l l}$. Left: sequential decay. Right: branched decay.

parameters are not a priori fixed. The sector of the supersymmetric parameter space characterized by these features is denoted as Scenario $\mathcal{B}$. Within this scenario it follows from Eqs. (4) that the following hierarchy holds for the coefficients $a_{i}^{(1)}$ of $\chi_{1}$ :

$$
\left|a_{1}^{(1)}\right|>>\left|a_{3}^{(1)}\right|,\left|a_{2}^{(1)}\right|,\left|a_{4}^{(1)}\right| .
$$

Table I summarizes the representative features of scenarios $\mathcal{A}$ and $\mathcal{B}$ to be used below for the definition of our benchmark.

In the present paper we focus our investigation of scenario $\mathcal{A}$, postponing the discussion of scenario $\mathcal{B}$ for a subsequent publication.

\section{SIGNALS AT THE LHC}

Squarks and gluinos are expected to be copiously produced in the $p p$ scattering processes at the LHC: $p p \rightarrow$ $\tilde{q} \tilde{q}, \tilde{q} \tilde{q}^{*}, \tilde{g} \tilde{g}, \tilde{q} \tilde{g}$. In turn, squarks, produced either directly or through gluinos, can generate the sequential decay chains:

$$
\tilde{q} \rightarrow q \chi_{i} \rightarrow q \tilde{f} f \rightarrow q \bar{f} f \chi_{1}
$$

and the branched ones:

$$
\tilde{q} \rightarrow q \chi_{i} \rightarrow q(Z, h, H, A) \chi_{1} \rightarrow q \bar{f} f \chi_{1},
$$

where $f$ stands for a fermion, $\bar{f}$ for its supersymmetric partner; from now on the neutralino subscript $i$ can only take the values 2, 3 or 4 . These two topologies are shown in Fig 1 .

These are the key processes to be studied at the LHC to measure the sfermions and neutralinos masses 33 38]. They would be characterized by hard jets, specific two-body decays and a transverse missing energy (under the hypothesis of R-parity conservation). The determination of the masses cannot proceed through a full reconstruction of the decay chains, since the LSP neutralino escapes detection, but rather by measurements of specific features in unidimensional and multidimensional distributions in kinematical variables.

Typical strategies for determining the sfermion and neutralino masses consist in: a) measurements of endpoints in single invariant mass distributions [33 38], b) correlations among different invariant mass distributions [39].

\section{A. General properties of the decay chains}

The decay chains (9)-(10) have in common the first step, i.e. the squark decay $\tilde{q} \rightarrow q \chi_{i}$, which can proceed either through gauge coupling (which involve the gaugino components of $\chi_{i}$ ), or Yukawa coupling (which involve the higgsino 
components of $\chi_{i}$ ). In the following we will assume for simplicity a situation where the gluino is decoupled, by taking $M_{3} \gg m_{\tilde{q}}$. In this case squarks can be only produced with the same flavor of the partons inside the protons which induce the hadronic processes at the LHC. As a consequence of this Yukawa couplings have a subdominant role as compared to the gauge couplings, since the relative importance of the Yukawa couplings to the gauge ones depends on the ratio $m_{q} / m_{Z}\left(m_{q}\right.$ and $m_{Z}$ being the quark mass and the Z-boson mass, respectively) and heavy flavors are scarce in the proton composition. In particular, in this case in the process $\tilde{q} \rightarrow q \chi_{i}$, the $\chi_{i}$ 's having a dominant gaugino composition are preferentially produced.

Sequential chains are differentiated from the branched chains by the features of the decay process undertaken by the intermediate neutralino state $\chi_{i}$. In the sequential chain the decay proceeds through the process: $\chi_{i} \rightarrow \tilde{f} f \rightarrow \bar{f} f \chi_{1}$ with a branching ratio $\operatorname{BR}\left(\chi_{i} \rightarrow \tilde{f} f \rightarrow \bar{f} f \chi_{1}\right)=\operatorname{BR}\left(\chi_{i} \rightarrow \tilde{f} f\right) \operatorname{BR}\left(\tilde{f} \rightarrow f \chi_{1}\right)$. In the following we will limit our considerations to the most interesting cases, where $f$ is a charged lepton (i.e. $f=l=e, \mu, \tau)$. The $\operatorname{size}$ of $\operatorname{BR}\left(\chi_{i} \rightarrow \tilde{l} l\right)$ depends sensitively on the $\chi_{i}$ composition. If $\chi_{i}$ is dominantly a gaugino, because of the universality of the gaugino couplings, the branching ratios $\mathrm{BR}\left(\chi_{i} \rightarrow \tilde{l} l\right)$ for the three lepton flavours are about the same ; if $\chi_{i}$ is dominantly a Higgsino, $\chi_{i}$ decays predominantly into a $\tilde{\tau} \tau$ pair.

In the branched chain, $\chi_{i}$ decays either through the Z-boson or through a Higgs boson. The first case, $i . \quad e$. $\chi_{i} \rightarrow Z+\chi_{1}$, involves only the Higgsino components of the two neutralino states; the $Z$ boson subsequently decays into all (kinematically possible) $\bar{f} f$ pairs according to the Standard Model branching fractions. The second case, $i$. e. $\chi_{i} \rightarrow(h, A, H)+\chi_{1}$, in order to have a sizable $\mathrm{BR}$, requires that one neutralino state is dominantly a gaugino, the other dominantly a Higgsino. Since in the scenarios considered in the present paper $\chi_{1}$ is dominantly a B-ino state, $\chi_{i} \rightarrow(h, A, H)+\chi_{1}$ is of interest when $\chi_{i}$ is dominated by the Higgsino components. Because of the hierarchical character of the Yukawa coupling, the subsequent decays of the Higgs bosons are dominated by the production of a $b$ $-\bar{b}$ pair.

A detailed discussion of the branching ratios for the various processes involved in the decay chains (9)-(10) for the LNM are given in Ref. [15].

\section{SELECTING A BENCHMARK IN THE LNM SCENARIO}

In the present paper we wish to address the following two points: i) Is the LNM scenario $\mathcal{A}$ (as defined in Table \) detectable at the LHC over the SM background? ii) Is it possible to kinematically reconstruct the neutralino mass at the LHC in its low range within the LNM scenario $\left(m_{\chi} \simeq 10 \mathrm{GeV}\right)$ ?

As discussed in Section IA , one of the basic features of the LNM scenarios is that some of the SUSY parameters are forced into rather strict intervals. As shown in Table प, this is particularly true in Scenario $\mathcal{A}$ for the parameters $M_{1}, \mu, \tan \beta$ and $m_{A}$, the latter three parameters being just beyond the LEP and Tevatron sensitivities.

A scatter plot of these 4 parameters where all the experimental constraints listed in Section II are implemented is given in Fig 22 here the parameters have been varied in the following narrow ranges: $10 \mathrm{GeV} \leq M_{1} \leq 14 \mathrm{GeV}$, $110 \mathrm{GeV} \leq \mu \leq 140 \mathrm{GeV}, 30 \leq \tan \beta \leq 40,90 \mathrm{GeV} \leq m_{A} \leq 105 \mathrm{GeV}$, in accord with the intervals of scenario $\mathcal{A}$ in Table I. As far as these parameters are concerned, the choice of a benchmark is quite restricted.

In Fig. 2 we plot with a triangular symbol the representative point that we adopt in Table $\Pi$ as our benchmark: $M_{1}=14 \mathrm{GeV}$ (corresponding to $m_{\chi} \simeq 11 \mathrm{GeV}$ ), $\mu=126 \mathrm{GeV}, \tan \beta=34$ and $m_{A}=97 \mathrm{GeV}$. The LNM scenario is basically independent on the remaining six parameters of the model $\left(M_{2}, M_{3}, m_{\tilde{q}}, m_{\tilde{t}}, m_{\tilde{l}}\right.$ and $\left.A\right)$, which are only constrained by the various experimental limits listed in Section [I] In particular LHC physics is very sensitive through the SUSY production cross section to the $m_{\tilde{q}}$ parameter (which drives the mass of squarks of the first two families corresponding to the flavors more abundant in colliding protons) and to the gluino mass $M_{3}$. The LHC early runs have already started to introduce constraints on these parameters, which, however, strongly depend on the adopted SUSY scenario [40 44]. Following the approach already adopted in Ref. 15], for simplicity in the present paper we will limit our discussion to the case in which the gluino is heavier than the squark, and for definiteness we will fix it at the representative value $M_{3}=2 \mathrm{TeV}$.

In Ref. [7] the possible impact of some early analyses by the CMS and ATLAS Collaborations at the LHC [40 42] on the LNM scenario was investigated. The data considered there consisted in the results of searches for supersymmetry in proton-proton collisions at a center-of-mass energy of $7 \mathrm{TeV}$ with an integrated luminosity of $35 \mathrm{pb}^{-1}$ [40], i.e. the results of the CMS Collaboration for events with jets and missing transverse energy [40], and those of the ATLAS Collaboration by studying final states containing jets, missing transverse energy, either with an isolated 

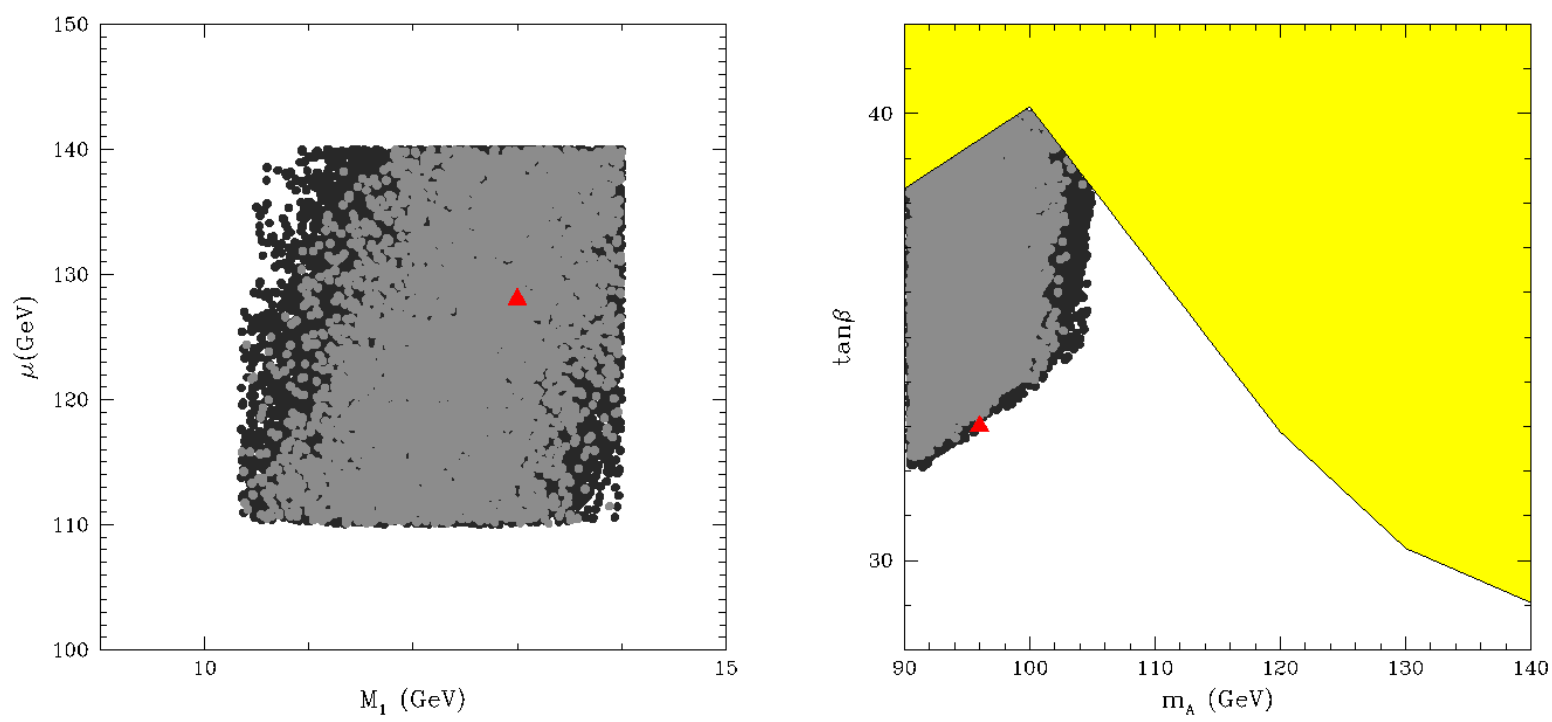

FIG. 2: Scatter plot of the parameters $M_{1}$ and $\mu$ (left) and of the parameters $m_{A}$ and $\tan \beta$ (right) in the LNM scenario. The dots, in black and in gray, correspond to the range of parameters given in the text in accord with scenario $\mathcal{A}$ of Table $\square$ the subset of dots in gray show the configurations which fall into region I of Fig. 4 allowing sequential decays through production and decay of a $\chi_{4}$ (see text). The (red) triangle shows the LNM-seq benchmark given in Table $\amalg$ that is analyzed in detail in the present paper. In the right-hand plot the yellow shaded area shows the region disallowed in the plane $\left(m_{A}-\tan \beta\right)$ from the results of Refs. [18], as derived in the analysis of Ref. [19].

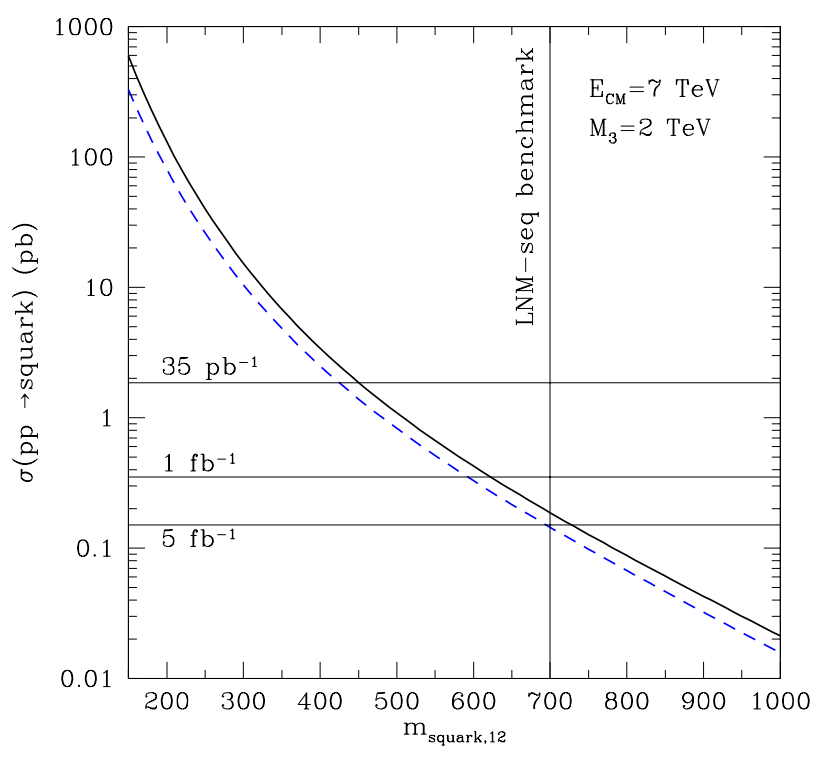

FIG. 3: Squark production cross section at the LHC as a function of the mass of the squarks of the first two families $m_{\text {squark }, 12} \simeq m_{\tilde{q}}$ for a center-of-mass energy $E_{C M}=7 \mathrm{TeV}$ and for a heavy gluino mass, $M_{3}=2 \mathrm{TeV}$. The solid line shows the next-to-leading order value calculated with Prospino [45] while the dashed line shows the same quantity at the leading order. The highest horizontal line marked " $35 \mathrm{pb}^{-1}$ " shows the bound on the production cross section from early analyses of CMS and ATLAS Collaborations at the LHC as calculated in Ref. [7] for the LNM scenario. The lower horizontal lines show an estimation for the sensitivity of the LHC run at $E_{C M}=7 \mathrm{TeV}$ for two different values of the collected luminosity. The vertical line at $m_{\text {squark }}=700 \mathrm{GeV}$ indicates the value of the parameter $m_{\tilde{q}} \simeq m_{\text {squark,12 }}$ that is adopted in the LNM-seq benchmark introduced in Table 


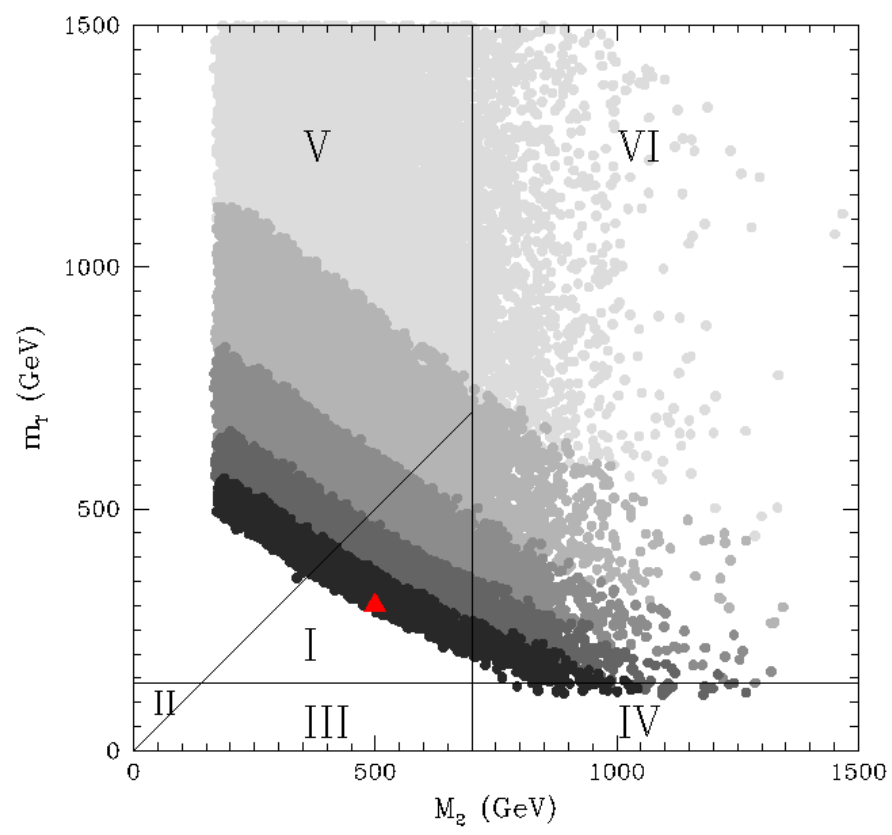

FIG. 4: Scatter plot of the parameters $M_{2}$ and $m_{\tilde{l}}$ in the LNM scenario with $m_{\tilde{q}}=700 \mathrm{GeV}$ and $M_{3}=2 \mathrm{TeV}$. All plotted configurations are allowed by the observational constraints summarized in Section III this plot the parameters $M_{1}, \mu$, tan $\beta$ and $m_{A}$ are sampled in the ranges of Scenario $\mathcal{A}$ indicated in Table $\square$ and their numerical scan is shown in Fig. 2 The remaining two parameters of the model, $A$ and $m_{\tilde{t}}$, are shown in Fig 5 . All the configurations are subject to the experimental bounds listed in Section [1] The points are plotted in 5 increasingly dark tones of gray corresponding to the following subintervals for the allowed range for the muon anomalous magnetic moment: $31<\Delta a_{\mu} \times 10^{11}<130,130<\Delta a_{\mu} \times 10^{11}<230$, $230<\Delta a_{\mu} \times 10^{11}<330,330<\Delta a_{\mu} \times 10^{11}<430$ and $430<\Delta a_{\mu} \times 10^{11}<479$. The regions indicated by numbers I, II, III, IV, $\mathrm{V}$ and VI correspond to different kinematic regimes for the sequential decay (see text for details). In particular in regions I,II III and IV sequential decays are kinematically accessible through a next-to-lightest neutralino $\chi_{i}$ with $i=4$ (region I), $i=3,4$ (region II), $i=2,3,4$ (region III) and $i=2,3$ (region IV). The triangular symbol indicates the LNM-seq benchmark given in Table III that is analyzed in detail in the following Sections.

lepton (electron or muon) [41] or without final leptons [42]. As reported in Refs. [40, 41] the data appeared to be consistent with the expected Standard Model (SM) backgrounds; thus an upper bound on the SUSY production cross section $\sigma\left(\mathrm{pp} \rightarrow\right.$ squark) at the LHC for a center-of-mass energy $E_{C M}=7 \mathrm{TeV}$ was derived in Ref. 7] for the LNM scenario. In Fig. 3 we plot this cross section as a function of the common mass of the squark of the first two families $m_{\text {squark } 12} \simeq m_{\tilde{q}}$ for $M_{3}=2 \mathrm{TeV}$. Moreover, the highest horizontal line marked " $35 \mathrm{pb}^{-1}$ " shows the upper bound on the same quantity as derived in Ref. [7], that implies a lower limit $m_{\tilde{q}} \gtrsim 450 \mathrm{GeV}$ when the gluino is heavy. In the same figure the lower horizontal lines show an estimation for the sensitivity of the LHC run at $E_{C M}=7 \mathrm{TeV}$ for two different values of the collected luminosity, $\mathcal{L}=1 \mathrm{fb}^{-1}$ and $\mathcal{L}=5 \mathrm{fb}^{-1}$, naively obtained by scaling down the bound on the cross section from [7] with the square root of the exposition. Assuming $\mathcal{L}=5 \mathrm{fb}^{-1}$ as the expected total collected exposition in the LHC run at $E_{C M}=7 \mathrm{TeV}$ before the stop scheduled for the end of the year 2011, one can see that a value $m_{\tilde{q}} \simeq 700 \mathrm{GeV}$ would be by that time just on the verge of discovery, possibly already providing a small excess over the standard model background. For this reason in the following we will adopt $m_{\tilde{q}}=700 \mathrm{GeV}$ as our benchmark value of the soft squark parameter for the first two families.

We proceed now to discuss the remaining 4 parameters, $M_{2}, m_{\tilde{l}}, m_{\tilde{t}}$ and $A$. As discussed in Section $\amalg \mathrm{A}$ the properties of the sequential decay of Eq. (9) that we wish to analyze depend sensitively on the hierarchy among the masses of the particles involved, i.e. on the masses of the squarks, of the next-to-lightest neutralino and of the slepton. In our scenario these three mass scales are driven by $m_{\tilde{q}}, m_{\tilde{l}}$ and $M_{2}$ or $\mu$, determining in particular whether the spectrum of neutralinos is normal or inverted and if the decay $\chi_{i=2,3,4} \rightarrow \tilde{l} l$ is kinematically allowed. Since we have fixed $m_{\tilde{q}}=700 \mathrm{GeV}$ and in Scenario $\mathcal{A}$ the $\mu$ parameter is constrained to the narrow range $110 \mathrm{GeV} \lesssim \mu \lesssim 140 \mathrm{GeV}$, in Fig. 4 we discuss this mass hierarchy in the plane of the remaining two parameters, $M_{2}$ and $m_{\tilde{l}}$. In this plane we 
schematically represent with a line at the constant value $m_{\tilde{l}}=140 \mathrm{GeV}$ the scale of the $\mu$ parameter. Then, with the exception of the narrow band where $M_{2} \simeq \mu$, one has $\mu \simeq m_{\chi_{2,3}}, M_{2} \simeq \chi_{4}$ when $M_{2}>\mu$ (normal hierarchy) and $\mu \simeq m_{\chi_{3,4}}, M_{2} \simeq m_{\chi_{2}}$ when $M_{2}>m u$ (inverted hierarchy). Moreover $m_{\tilde{l}}$ fixes the scale of the slepton masses. This implies that schematically one can divide the $M_{2}-m_{\tilde{l}}$ plane in six regions:

- Region I $\left(M_{2}>m_{\tilde{l}}>\mu\right)$. Here only the decay $\chi_{4} \rightarrow \tilde{l} l$ is kinematically allowed, with $m_{\chi_{4}} \simeq M_{2}$.

- Region II $\left(\mu>m_{\tilde{l}}>M_{2}\right)$. Here only the decays $\chi_{3,4} \rightarrow \tilde{l l}$ are kinematically allowed, with $m_{\chi_{3,4}} \simeq \mu$;

- Region III $\left(M_{2}>\mu>m_{\tilde{l}}\right)$. All decays $\chi_{2,3,4} \rightarrow \tilde{l l}$ are kinematically allowed,

- Region IV $\left(M_{2}>m_{\tilde{q}}=700 \mathrm{GeV}, \mu>m_{\tilde{l}}\right)$. Here only the decays $\chi_{2,3} \rightarrow \tilde{l l}$ are kinematically allowed, with $m_{\chi_{2,3}} \simeq \mu$

- Regions V and VI. No sequential decays are kinematically allowed.

In the same figure the scatter plot represents a scan of the LNM parameter space with $110 \mathrm{GeV}<M_{2}<1500 \mathrm{GeV}$, $110 \mathrm{GeV}<m_{\tilde{l}}<1500 \mathrm{GeV}$, while the other parameters are in the ranges of Scenario $\mathcal{A}$ given in Table 【 The points are plotted in 5 increasingly dark tones of gray corresponding to the following sub-intervals for the allowed range for the muon anomalous magnetic moment: $31<\Delta a_{\mu} \times 10^{11}<130,130<\Delta a_{\mu} \times 10^{11}<230,230<\Delta a_{\mu} \times 10^{11}<330$, $330<\Delta a_{\mu} \times 10^{11}<430$ and $430<\Delta a_{\mu} \times 10^{11}<480$. In this way it is possible to see that the kinematic regions II and III (at least for the particular choice of $m_{\tilde{q}}$ adopted here) are not allowed by the upper bound on $\Delta a_{\mu}{ }^{1}$. This restricts the present discussion to the possibility of having a sequential decays only in regions I and IV. In region IV sequential decays proceed through production and decays of very light next-to-lightest neutralinos $\chi_{2,3}$ with $m_{\tilde{l}} \lesssim m_{\chi_{12}} \simeq \mu \lesssim 140 \mathrm{GeV}$ that, according to the discussion of Section II are of higgsino type. These features make the detection of sequential decays in this case quite challenging, since the corresponding branching ratio is suppressed both by the small available phase space, and by the fact that, due to their higgsino nature, a large fraction of the $\chi_{2,3}$ particles decay through the branched topology to a $Z$ or a Higgs boson (see Fig. 1 right). Moreover, the higgsino nature of the $\chi_{2,3}$ particles also implies that when a sequential decay actually takes place it mainly proceeds to tau final states that are more difficult to measure compared to muons and electrons. As a consequence of this, for the choice of a benchmark for our discussion of sequential decays we decide to focus on the only remaining possibility, i.e. region I. In Figs. 2 and 5 the configurations belonging to region 1 are plotted in grey.

Region I looks more promising than region IV for sequential decays. In fact in this case decays proceed through a $\chi_{4}$ with $m_{\chi_{4}} \simeq M_{2}$ which is of Wino type implying a smaller coupling to the $Z$ and Higgs bosons which reduces branched decays and leading to comparable signals to electrons, muons and taus in sequential ones. Moreover, depending on the choice of the $M_{2}$ and $m_{\tilde{l}}$ parameters, the phase space available to both decays $\tilde{q} \rightarrow q \chi_{4}$ and $\chi_{4} \rightarrow l \tilde{l}$, can be sizeable. As the scatter plot of Figure 4 shows, this still allows for a wide range of possibilities. With the spirit of choosing light values for both the $\chi_{4}$ and the sleptons masses and to maximize at the same time the phase space available to the decay, in Table $\amalg$ we choose as our benchmark the values $M_{2}=500 \mathrm{GeV}$ and $m_{\tilde{l}}=300 \mathrm{GeV}$, corresponding to the point shown in Fig. 4 with a triangular symbol lying somewhat in the center of the triangle of region I and close to the lower bound on both parameters from the muon anomalous magnetic moment.

We conclude the present discussion with the last two parameters, $A$ and $m_{\tilde{t}}$. Apparently, since they do not affect directly either the neutralino relic abundance in the LNM scenario or the sequential decays we wish to discuss, pinning down a value for these two parameters may seem quite arbitrary. However, as discussed in Ref. 7], the combination of the experimental constraints from the $b \rightarrow s \gamma$ and the $B \rightarrow \tau \nu$ decays may induce a strong correlation between A and $m_{\tilde{t}}$, restricting their range of variation. This is shown in Fig 5 where a scatter plot of these two parameters is given and both constraints are applied. Notice that the correlation is further enhanced if configurations plotted in

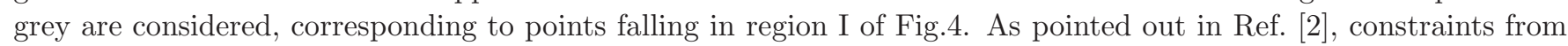
rare B-meson decays are affected by uncertainties both in experimental measurements and theoretical estimates, so should be considered with care. In particular when the bound from the $B \rightarrow \tau \nu$ is not implemented the correlation

\footnotetext{
1 The quantity $\Delta a_{\mu}$ does not depend on the SUSY hadronic sector, so a change in the $m_{\tilde{q}}$ parameter would imply only a shift of the vertical line separating kinematic regions I, III and V from regions IV and VI, without modifying the scatter plot. This implies that for $m_{\tilde{q}} \gtrsim 800 \mathrm{GeV}$ the kinematic region III would be allowed for sequential decays
} 


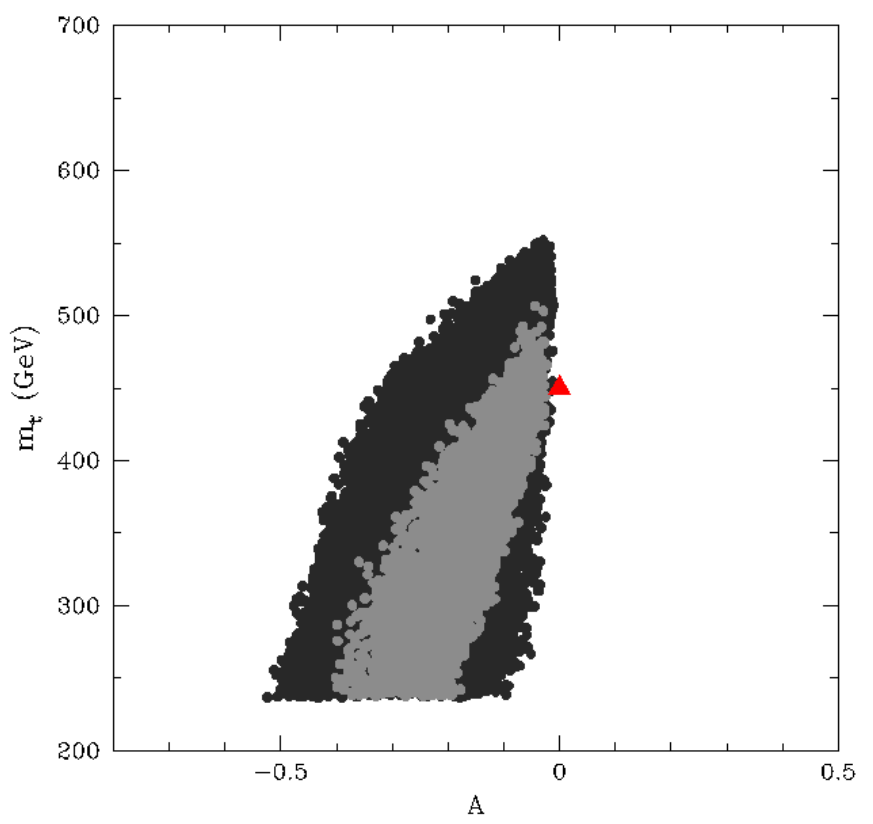

FIG. 5: Scatter plot of the parameters $A$ and $m_{\tilde{t}}$ in the LNM scenario. The color code is the same as in Figure 2 triangle shows the LNM-seq benchmark given in Table I

\begin{tabular}{|c|c|c|c|c|c|c|c|c|c|c|}
\hline benchmark & $M_{1}[\mathrm{GeV}]$ & $M_{2}[\mathrm{GeV}]$ & $M_{3}[\mathrm{GeV}]$ & $\mu[\mathrm{GeV}]$ & $\tan \beta$ & $m_{A}[\mathrm{GeV}]$ & $m_{\tilde{l}}[\mathrm{GeV}]$ & $m_{\tilde{q}}[\mathrm{GeV}]$ & $m_{\tilde{t}}[\mathrm{GeV}]$ & $A$ \\
\hline \hline LNM-seq & 14 & 500 & 2000 & 126 & 34 & 97 & 300 & 700 & 444 & -0.08 \\
\hline
\end{tabular}

TABLE II: The LNM-seq benchmark analyzed in the present paper.

between $A$ and $m_{\tilde{t}}$ is no longer present [7]. With this caveat, and for the sake of definiteness, we may choose our benchmark as a configuration within the grey region of Fig. 5 In particular, in order to kinematically suppress the decay $\chi_{4} \rightarrow t \tilde{t}$ and maximize the leptonic sequential signature, we choose as our benchmark a configuration with a value of $m_{\tilde{t}}$ close to the upper edge of the allowed range. Our final choice for the last two benchmark parameters is plotted in Fig 5 with the triangular symbol, and corresponds to the values given in Table II. $A=-0.08$ and $m_{\tilde{t}}=444$ $\mathrm{GeV}$.

\section{EARLY DISCOVERY OF LIGHT NEUTRALINOS AT THE LHC}

The LHC has already started to put bounds on the supersymmetric parameter space. In particular, the very constrained SUGRA scenario, in which soft masses and the trilinear coupling are all unified at the GUT scale and the $\mu$ and $m_{A}$ parameters are predicted by radiative elecroweak symmetry breaking, appears already to be disfavored by the data 40 44]. Actually, in this scenario lower bounds on both gluino and squark masses are already close to the $\mathrm{TeV}$ range, in tension with the naturalness picture that is considered one of the motivations of SUSY in the first place.

As already mentioned in the previous section, in order to discuss LHC bounds in the LNM scenario, a dedicated analysis was performed in Ref. 7] for some of the specific signatures searched by ATLAS and CMS (namely jets+missing transverse energy and one isolated lepton or jets + missing transverse energy and no leptons). As discussed in that paper, if squark soft masses of the three families are assumed to be degenerate, the combination of the ensuing LHC constraints on squark and gluino masses with the experimental limit on the $b \rightarrow s+\gamma$ decay imply a lower bound on the neutralino mass $m_{\chi}$ that can reach the value of $11.9 \mathrm{GeV}$ when the gluino mass is at its lower bound, but is essentially unchanged for a heavy gluino. However this bound on $m_{\chi}$ is no longer in place when, as in the present 
analysis, the universality condition among squark soft parameters is relaxed. This implies that for non-universal squark masses the lower bound on the neutralino mass remains at the value $9 \mathrm{GeV}$ mentioned in the Introduction.

The LHC is expected to collect $\mathcal{L} \simeq 5 \mathrm{fb}^{-1}$ of integrated luminosity at the end of the 2011 run at a center-of-mass energy of $7 \mathrm{TeV}$. In order to estimate the expected signal at the end of the $7 \mathrm{TeV}$ run of the LHC for the LNMseq benchmark introduced in the previous Section we have used ISAJET [46], applying the same kinematic cuts as described in Ref. [40] for the early discovery signature of jets + missing transverse energy and no leptons. The result of the simulation is 260 events from SUSY compared to 133 events expected from backgrounds. The background estimation is an extrapolation based on CMS measurements. The expected number of SUSY events $N$ is related to the SUSY production cross section $\sigma \equiv \sigma(\mathrm{pp} \rightarrow$ squark) and to the luminosity $\mathcal{L}$ by the relation $N=\epsilon \times \mathcal{L} \times \sigma$, where $\epsilon$ is the total efficiency due to selection cuts, that for the LNM-seq benchmark we estimate $\epsilon=0.21$. So, in agreement to the discussion of the previous Section, the LNM-seq benchmark is expected to provide a slight excess over the background, namely at the level of a $\simeq 3.2 \sigma$ significance assuming that our estimation on the background has a $5 \%$ relative uncertainty.

Such an early hint of SUSY in the $7 \mathrm{TeV}$ run of the LHC would not, however, allow to draw any conclusions on the mass and properties of the neutralino, let alone whether the observed excess is compatible to an LNM scenario or not. In fact, since the neutralino escapes undetected, its mass can only be reconstructed by observing the sequential decay introduced in Eq. (9) where the observed fermions are either muons or electrons in order to have a better discrimination of the signal over the hadronic background. In the corresponding final state of 2 jets + missing transverse energy and 2 isolated leptons we estimate from the above simulation $\simeq 2$ signal events at $\mathcal{L}=5 \mathrm{fb}^{-1}$ (corresponding to an efficiency $\epsilon=0.00155$ ) obviously insufficient to get any information about the masses. For this kind of analysis $E_{c m}=14 \mathrm{TeV}$ and a higher collected luminosity will be needed.

\section{LNM SPECTROSCOPY AT THE LHC WITH $E_{C M}=14$ TEV}

The use of kinematic endpoints to reconstruct the mass spectrum in a sequential decay chain where the lightest particle escapes detection and with the topology as shown in Fig 1 1has been widely discussed in the literature 36, 38, 39, 47]. This technique is based on the simple idea of reconstructing the four unknown masses of the problem $\left(m_{\chi}, m_{\tilde{l}}, m_{\chi_{i}}, m_{\tilde{q}}\right)$ by inverting the four observable kinematic endpoints $\left(m_{\ell \ell}^{\max }, m_{j \ell \ell}^{\max }, m_{j \ell(l o)}^{\max }, m_{j \ell(h i)}^{\max }\right)$, where $j$ indicates the jet produced by the emitted quark and $m_{j \ell(l o)} \equiv \operatorname{Min}\left(m_{j \ell_{n}}, m_{j \ell_{f}}\right), m_{j \ell(h i)} \equiv \operatorname{Max}\left(m_{j \ell_{n}}, m_{j \ell_{f}}\right)$ are defined in order to remove the ambiguity between the near and the far leptons $\ell_{n}, \ell_{f}$ (shown in Fig 1) which are not experimentally distinguishable. The expected value of such endpoints are given in Table III for the LNM-seq benchmark introduced in Section IV. For completeness we give the analytic expressions of the endpoints as a function of the masses in Appendix A

Two problems however arise in this apparently straightforward procedure. The first issue is related to the fact that for particular mass combinations in the sequential decay the four aforementioned endpoints are not independent, since the following relation holds [38]:

$$
\left(m_{j \ell \ell}^{\max }\right)^{2}=\left(m_{\ell \ell}^{\max }\right)^{2}+\left(m_{j \ell(h i)}^{\max }\right)^{2}
$$

In particular, this is true whenever $m_{\chi}<m_{\tilde{l}}^{2} / m_{\tilde{q}}$. Taking into account the experimental constraint $m_{\tilde{l}} \gtrsim 100$ $\mathrm{GeV}$ implies that for a light neutralino of mass $m_{\chi}=10 \mathrm{GeV}$ the correlation (11) is verified if $m_{\tilde{q}} \lesssim 1 \mathrm{TeV}$ and, in particular, holds in the LNM-seq benchmark. To compensate for the occurrence of only three independent variables of the physical masses instead of four an additional measurement is needed, for instance the lower kinematic endpoint $m_{j \ell \ell(\theta>\pi / 2)}^{\min }$ introduced in Ref. [36], which corresponds to the lower bound of the $m_{j \ell \ell}$ histogram with the additional constraint $\left(m_{\ell \ell}^{\max }\right)^{2} / 2<\left(m_{\ell \ell}\right)^{2}<\left(m_{\ell \ell}^{\max }\right)^{2}$.

The second issue related to the analysis of endpoints is that when Eq. (11) is verified the inversion procedure is known to have multiple solutions, so that the determination of the masses $\left(m_{\chi}, m_{\tilde{l}}, m_{\chi_{i}}, m_{\tilde{q}}\right)$ is non-unique. This is indeed what happens in the LNM-seq benchmark, as shown in Table III. In the top part of the Table we report the values for the masses $\left(m_{\chi}, m_{\tilde{l}}, m_{\chi_{i}}, m_{\tilde{q}}\right)$. In the middle part of the same Table we give the expected endpoints of the kinematic variables $\left(m_{\ell \ell}, m_{j \ell \ell}, m_{j \ell(l o)}, m_{j \ell(h i)}, m_{j \ell \ell(\theta>\pi / 2)}^{\min }\right)$ in sequential decays, evaluated by using the expressions summarized in Appendix A] By using mass-inversion formulas [39] one finds that starting from the set of the endpoint values displayed in the Table one recovers the input set of mass values together with a second set of masses. In the Table such additional mass spectrum, hereafter referred as the duplicate of LNM-seq, is denoted as LNM-seq'. As 


\begin{tabular}{|c||c|c||}
\hline Variable & LNM-seq & LNM-seq' \\
\hline$m_{\chi}$ & 11 & 263 \\
$m_{\tilde{l}}$ & 305 & 383 \\
$m_{\chi_{i}}$ & 515 & 688 \\
$m_{\tilde{q}}$ & 703 & 896 \\
\hline \hline$m_{\ell \ell}^{\max }$ & $415(417.5 \pm 3.5)$ \\
$m_{j \ell \ell}^{\max }$ & $632(631.3 \pm 3.8)$ \\
$m_{j \ell(l o)}^{\max }$ & $338(342.2 \pm 4.3)$ \\
$m_{j \ell(h i)}^{\max }$ & $477(483 \pm 14)$ \\
$m_{j \ell \ell(\theta>\pi / 2)}^{\min }$ & $400(399.3 \pm 1.7)$ \\
\hline \hline$n^{\prime}, p^{\prime}$ & 282,385 & 232,477 \\
\hline
\end{tabular}

TABLE III: Expected endpoints (in GeV) of the kinematic variables $\left(m_{\ell \ell}, m_{j \ell \ell}, m_{j \ell(l o)}, m_{j \ell(h i)}, m_{j \ell \ell(\theta>\pi / 2)}^{\min }\right)$ for the LNM-seq benchmark of Table II] calculated using the expressions summarized in Appendix A In parenthesis we give measurements of the same quantities analyzing the output of a simulation of proton-proton collisions at $E_{C M}=14 \mathrm{TeV}$ assuming an integrated luminosity of $100 \mathrm{fb}^{-1}$ (see text). The benchmark LNM-seq' indicates the duplicate model of LNM-seq, i.e. a different mass pattern providing the same values of the observed endpoints in one-dimensional distributions [39]. In the last line the coordinates of the point $\left(m_{j \ell(l o)}, m_{j \ell(h i)}\right)=\left(n^{\prime}, p^{\prime}\right)$ can break the degeneracy between the two duplicated models. For this particular mass pattern it is not possible to measure the exact values $n^{\prime}, p^{\prime}$, since they lie on a straight boundary of the two-dimensional distribution (see Fig. 9(a) . However this is sufficient to break the degeneracy (see text). For this reason we do not provide a measured value of $n^{\prime}, p^{\prime}$ from the simulation. The analytic expressions of $\left(n^{\prime}, p^{\prime}\right)$ as a function of the physical masses are given in Appendix $\mathrm{A}$

will be discussed in the following, in order to overcome this duplication problem it is necessary to go beyond endpoints in one-dimensional histograms and to analyze the correlations among different invariant masses in two-dimensional plots [39].

We wish now to discuss if the procedure outlined above can be applicable to determine the mass spectrum of the LNM-seq benchmark using the LHC data at $E_{C M}=14 \mathrm{TeV}$. In order to do this, we simulate proton-proton collisions at $E_{C M}=14 \mathrm{TeV}$ using ISAJET and select events with two jets, two isolated leptons and missing transverse energy.

Notice that fast detector simulation tools which have been developed for the study of specific Supersymmetric scenarios such as in Supergravity-inspired (SUGRA) benchmarks are not available for the model under consideration here. So, in order to take into account the detector response, and specifically the uncertainty in the reconstruction of jet energies, in our simulation we smear the energy $E$ of quarks and gluons in the final state. In particular, we apply a resolution which depends on energy as $0.9 \sqrt{E}$. The Missing Transverse Energy (MET) is then determined by the vector sum of the energies of the neutrinos and the LSP plus any smearing applied to the hadronic jets. Due to the large mass of squarks and gluinos the dominant background is expected to be due to $t \bar{t}$ production. In particular, as shown below, large cut values are needed for an effective separation between the signal and the background. We apply to the output of our simulation the following cuts:

- The two leptons $\left(e^{+} e^{-}\right.$or $\left.\mu^{+} \mu^{-}\right)$are required to satisfy $|\eta|<2.4$ (where $\eta \equiv-\ln [\tan (\theta / 2)]$ is the pseudorapidity and $\theta$ is the angle with the beam axis) and $p_{T}>20 \mathrm{GeV}$ (where $p_{T}$ is the transverse momentum).

- The kinematic separation between outgoing states is required to be $\Delta R>0.5$, where $\Delta R \equiv \sqrt{(\Delta \eta)^{2}+(\Delta \phi)^{2}}$ and $\phi$ is the azimuthal angle.

- A missing transverse energy $E_{T}>300 \mathrm{GeV}$ is required, in order to indicate the presence of high energy neutralinos.

- The scalar sum of the transverse momenta $p_{T}$ of leptons and jets is required to be larger than $600 \mathrm{GeV}$.

- In the study of 2-dimensional distributions we have removed events where the invariant mass of the two outgoing leptons falls in the range $87 \mathrm{GeV}<M_{\ell \ell}<97 \mathrm{GeV}$, in order to subtract $Z$ boson decays. These events include also those produced in the branched decays shown in Fig 1 right. 

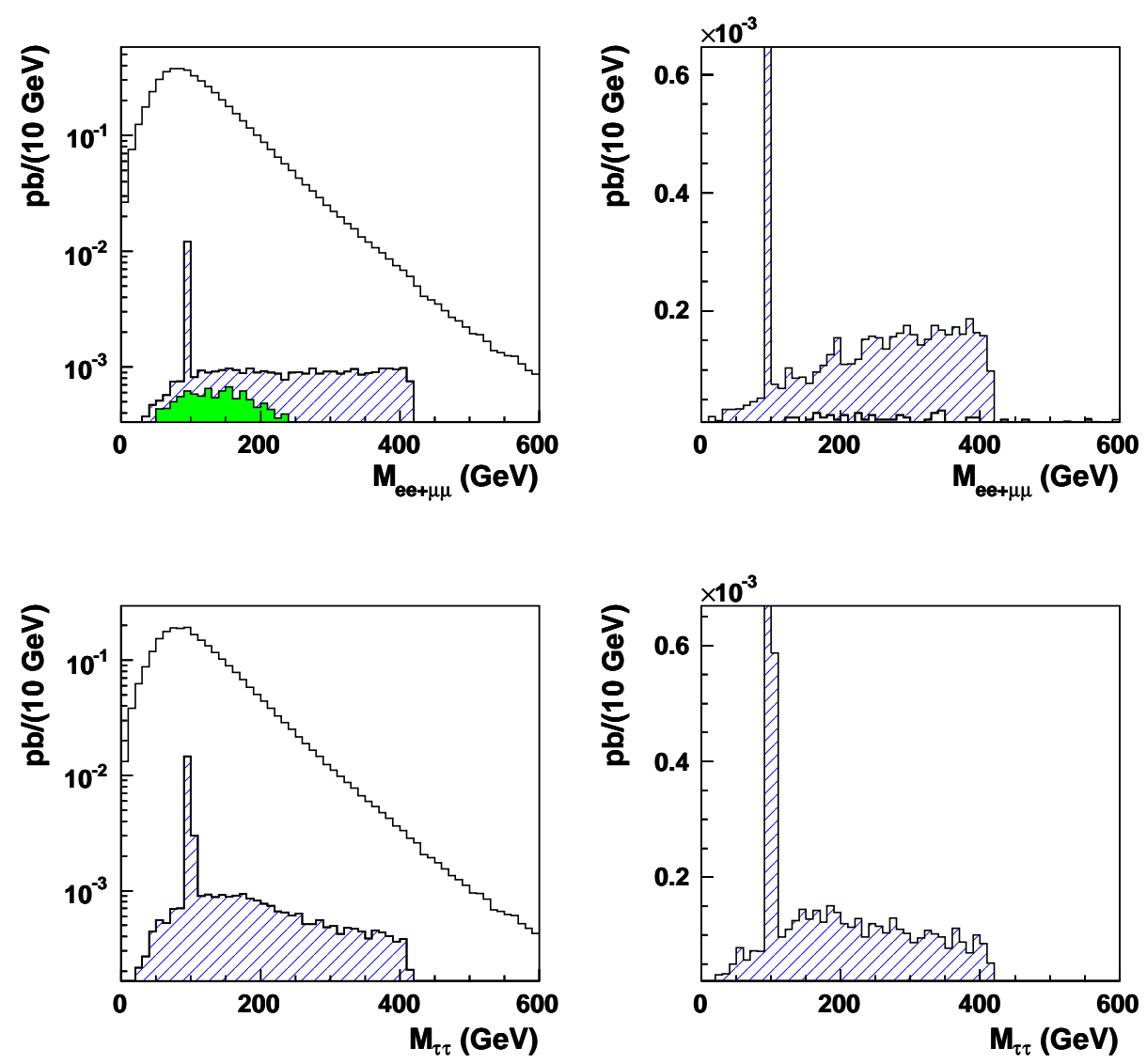

FIG. 6: Event distribution as a function of the final leptons invariant mass $m_{\ell \ell}$ for the benchmark LNM-seq. Plots on the left show the dilepton invariant mass at the preselection level while plots on the right show the same histograms after the cuts described in Section VI are applied, with the exception of the subtraction of the $Z$ peak (which is clearly visible). Upper plots show the case when the final leptons are given by $e^{+} e^{-}$and $\mu^{+} \mu^{-}$, while lower plots show the $\tau^{+} \tau^{-}$final state. In the plot on the upper left the hatched histogram shows the distribution of the $e \bar{e}+\mu \bar{\mu}$ events, while the (green) solid histogram shows the same for the $e \bar{\mu}+\mu \bar{e}$ events, which provides an estimation of the expected contribution from the SUSY background (see text). The shaded histogram in the plot on the upper right shows the background-subtracted distribution given by the difference between the $e \bar{e}+\mu \bar{\mu}$ and the $e \bar{\mu}+\mu \bar{e}$ histograms. In the case of the lower plots the hatched histogram on the left shows the distribution of $\tau^{+} \tau^{-}$events before cuts, while that on the right shows the same quantity after cuts. In all plots the white histogram shows the $t \bar{t}$ backgrounds.

Events with more than two jets or two leptons are rejected to minimize the effects of combinatorics. Out of the two jets in the event, only one must be associated to the dilepton in order to construct the $m_{j \ell(l o)}, m_{j \ell(h i)}$ invariant masses. The jet-dilepton pairing is found by choosing the combination that yields the smallest value for the $m_{\ell \ell j}$ invariant mass.

The expected distribution of the events with 2 jets +2 leptons + missing transverse energy produced in the simulation is shown in Fig 6 as a function of the invariant mass $m_{\ell \ell}$ and in Fig 7 as a function of $m_{\ell \ell j}$. In both Figures the plots on the left show events at the pre-selection level, while those on the right show the same distributions after the cuts described above, with the exception of the subtraction of the $Z$ peak. Moreover, upper plots show the case when the final leptons are electrons or muons, while lower plots show the $\tau^{+} \tau^{-}$final state. In all plots the white histogram shows the $t \bar{t}$ backgrounds, which, as can be seen, is strongly suppressed by the cuts.

A different and potentially sizeable source of background making the determination of endpoints difficult is also represented by SUSY events where the two charged leptons used to calculate the invariant mass are not originated in a sequential decay, but are produced instead by the decays of charginos originating from different decay chains. In the upper-right plots of Figs. 6 and 7 these undesired events are subtracted exploiting the fact that in this case 

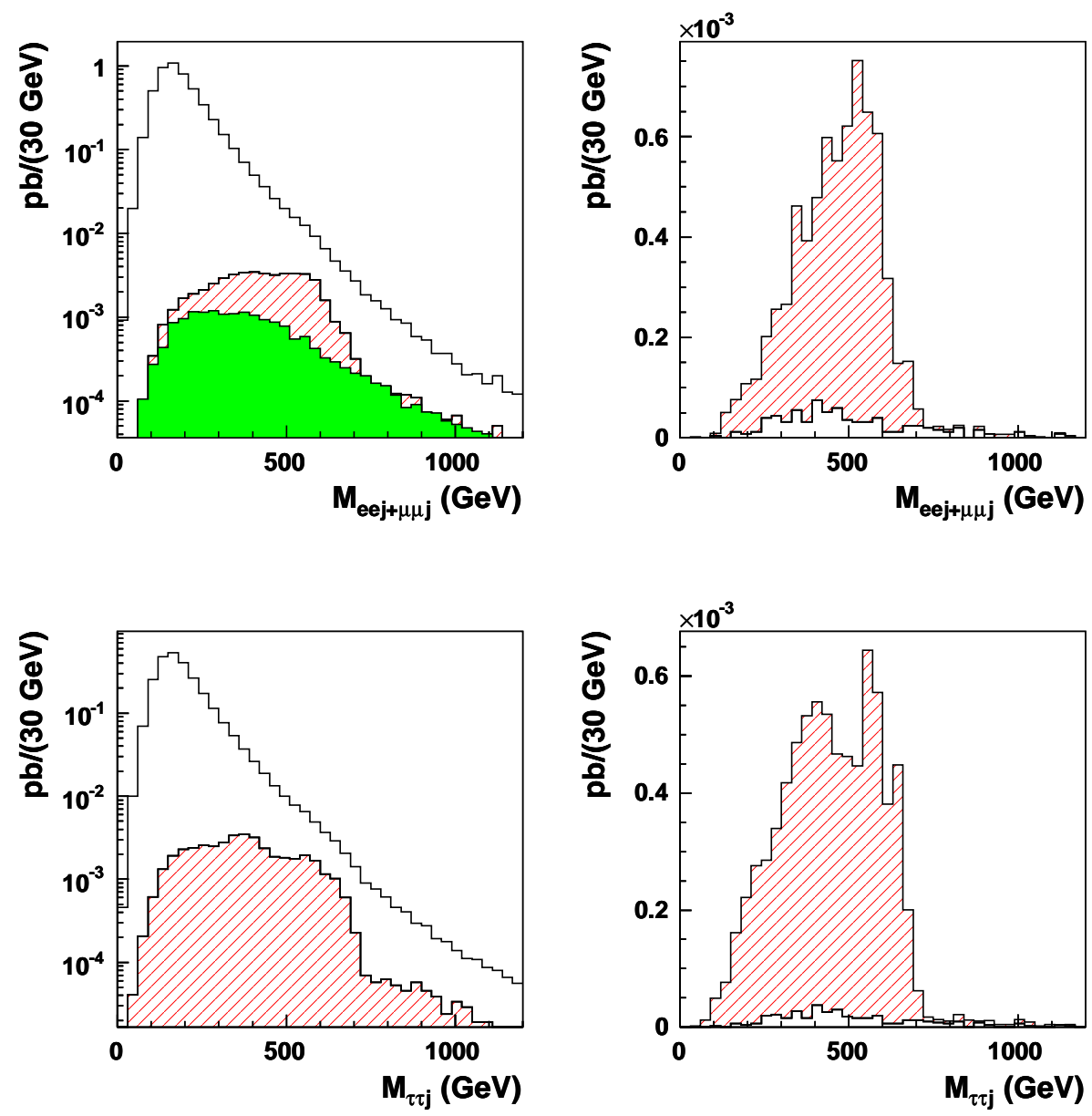

FIG. 7: Event distribution as a function of the invariant mass $m_{\ell \ell j}$ of the final leptons and the jet for the benchmark LNM-seq. The color code and the cuts are the same as in Fig 6 .

the flavors of the two leptons is uncorrelated, while when the two leptons are produced in the same sequential decay they have the same flavor. For this reason an effective subtraction of this background is obtained by taking the difference between the number of $e \bar{e}+\mu \bar{\mu}$ same-flavor events minus the number of events where the flavor of the final leptons is different, $e \bar{\mu}+\mu \bar{e}$. This is indeed an effective technique to subtract chargino decays and to allow a better identification of the endpoints, since the difference of the two distributions is expected to drop beyond the boundaries of the sequential process. In particular, in the plots on the upper left of Figs 6 and 7 the hatched histogram shows the distribution of the $e \bar{e}+\mu \bar{\mu}$ events while the solid histogram shows the same for the $e \bar{\mu}+\mu \bar{e}$ events, which provides an estimation of the expected contribution from the SUSY background. Moreover, the shaded histogram in the plots on the upper right show the background-subtracted distribution given by the difference between the $e \bar{e}+\mu \bar{\mu}$ and the $e \bar{\mu}+\mu \bar{e}$ histograms. In the case of the lower plots the hatched histogram on the left shows the distribution of $\tau^{+} \tau^{-}$events before cuts, while that on the right the same quantity after cuts. Comparison of left-hand figures and right-hand ones prove the overall effectiveness of the applied cuts to subtract the standard model background, in particular from top decays.

Notice that Figs. 6 and 7 are normalized to the luminosity and represent the theoretical expectations of the corresponding distributions. They are obtained using an integrated luminosity of $546 \mathrm{fb}^{-1}$ (or 3940 events after selection), allowing in particular to determine easily the position of the endpoints (that agree with the values given in Table III). However, for a lower value of the integrated luminosity the position of the endpoints is blurred by statistical fluctuations, worsening their determination. In the following we will assume for an optimistic and yet realistic prediction of the latter quantity $\mathcal{L}=100 \mathrm{fb}^{-1}$ at the end of the $14 \mathrm{TeV}$ LHC run. The corresponding prediction 
for the $m_{\ell \ell}$ distribution for a simulated experiment is given in Fig. 8(a) where the $e \bar{e}+\mu \bar{\mu}-e \bar{\mu}-\mu \bar{e}$ subtraction between same-flavor and different-flavor events has been applied to reduce the SUSY background from chargino decays. After selection cuts but without subtracting the $Z$ peak this plot contains 726 events, which become 502 when the $Z$ peak is subtracted.

The endpoint of this histogram provides the first edge $m_{\ell \ell}^{\max }$ needed for the kinematic reconstruction of the masses. In order to find it we employ a method inspired by an edge-detection algorithm frequently used in the field of image processing and computer vision [48]. Actually, the most sensitive method for measuring the position of an edge would be to obtain its expected distribution from a simulation and to perform a likelihood fit to the data. However, this method depends on SUSY parameters, hence lacking generality. On the other hand the edge-detection algorithm, while it may not yield the best sensitivity, has sufficient generality to be applied to a wider range of problems.

The edge-detection algorithm is a method that allows to find the endpoint of a sharply falling distribution by filtering the data histogram through an appropriate function. For concreteness, we take as a filtering function $f(x, \mu, \sigma)=$ $2 \sinh ((x-\mu) / \sigma) / \cosh ^{3}((x-\mu) / \sigma)$ and try to minimize the quantity:

$$
F(\mu, \sigma)=\sum_{i=1}^{N_{\text {data }}} f\left(x_{i}, \mu, \sigma\right),
$$

with respect to $\mu$ and with $\sigma$ fixed. In Eq.(12) $x_{i}$ represents the data count in the $i$-th bin of the histogram. The width parameter $\sigma$ has the effect of smoothing the distribution, hence making the algorithm immune to noise. The choice of $\sigma$ is determined by looking at the width of the distribution. If the value is too large, the edge determination is imprecise, whereas if the value is too small, then it will be sensitive to outliers.

In Fig. 8(b) we apply the method outlined above to filter the $m_{\ell \ell}$ histogram of Fig.8(a), We assume $\sigma=30 \mathrm{GeV}$ and the endpoint position is represented by the rightmost minimum, and is reported on the data histogram in $8(\mathrm{a})$ with a vertical solid line. In order to estimate the statistical fluctuation of $m_{\ell \ell}^{\max }$ we then repeat the same procedure for 100 pseudo-experiments identical to the one analyzed in Figs. 8(a) and 8(b). The corresponding frequency histogram for the outcome of $m_{\ell \ell}^{\max }$ is given in Fig $8(\mathrm{c})$. In this way we find $m_{\ell \ell}^{\max }=417.5 \pm 3.5$ (this value is reported in parenthesis in Table 【II).

In order to find the other endpoints needed to reconstruct the masses, in principle the above procedure can be applied also to the histograms obtained by plotting the same simulated events as a function of the other invariant masses $m_{\ell \ell j}$, $m_{j \ell(h i)}$ and $m_{j \ell(l o)}$. However, in the latter distributions the position of the endpoints cannot be determined accurately because the number of events is not large enough to saturate the endpoint of the histogram, which systematically drops at a value considerably lower than the true one for a lack of points in the tail. In this case an unambiguous determination of the endpoint is strictly speaking impossible, and only some educated guess can be made. In order to do this it can be useful to resort to two-dimensional plots. This is done in Figs $9(\mathrm{a})$ and 10(a) where the events of the pseudo-experiment plotted in Fig $8(\mathrm{a})$, and that lie to the left of the determined value of $m_{\ell \ell}^{\max }$, are plotted in the planes $m_{j \ell(l o)}-m_{j \ell(h i)}$ and $m_{\ell \ell}-m_{j \ell \ell}$. In this way both plots contain 497 events.

In the case of the $m_{j \ell(l o)}-m_{j \ell(h i)}$ two-dimensional plot of Fig. 9(a) the shape of the region covered by the data points nicely fits an isosceles triangle. This very symmetric shape is expected in two situations [39]: i) if the slepton is produced off-shell (i.e. if $m_{\tilde{l}}>m_{\chi_{i}}$ ), since in that case there is no longer distinction between the near and far lepton and the two leptons have exactly the same kinematic properties; ii) when the following relation among masses holds:

$$
\frac{m_{\chi}^{2}}{m_{\tilde{l}}^{2}}<\frac{m_{\tilde{l}}^{2}}{m_{\chi_{i}}^{2}}<\frac{1}{2-m_{\chi}^{2} / m_{\tilde{l}}^{2}},
$$

and at the same time there is a large hierarchy between the slepton mass and the neutralino, $m_{\chi} / m_{\tilde{l}} \ll 1$. In the latter case, that corresponds to our LNM-seq benchmark, the expected boundary is actually delimited by four vertexes, but the fourth point $\left(n^{\prime}, p^{\prime}\right)$, that is supposed to be used to break the degeneracy among duplicate models and whose coordinates are given in Table III, lies on the straight line $n^{\prime}+p^{\prime}=\left(m_{j \ell \ell} \max \right)^{2}$ when $m_{\chi} \ll m_{\tilde{l}}$ (it is represented by one of the two filled circles in Fig 9(a) and cannot be observed ${ }^{2}$. Notice however that, in spite of this, the degeneracy

\footnotetext{
${ }^{2}$ The density of points of the two-dimensional distribution is expected to have a step-like drop for $m_{j \ell(l o)}>p^{\prime}[\underline{39}$, allowing in principle
} 


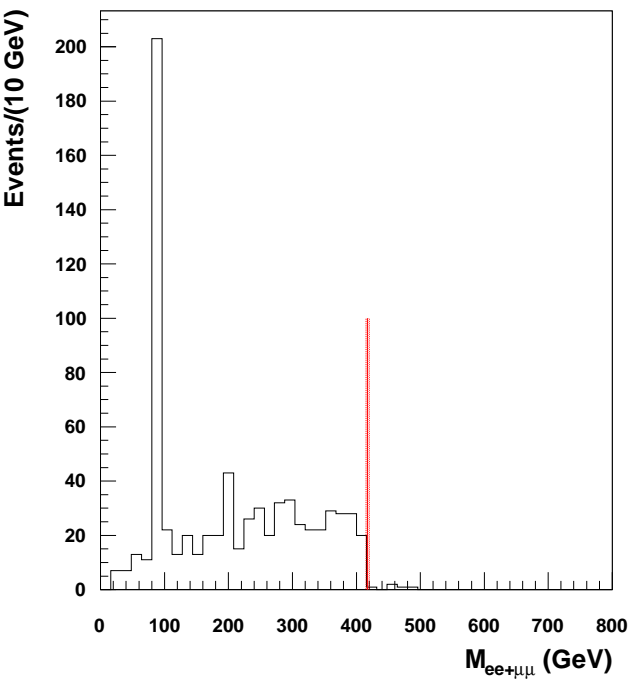

(a)

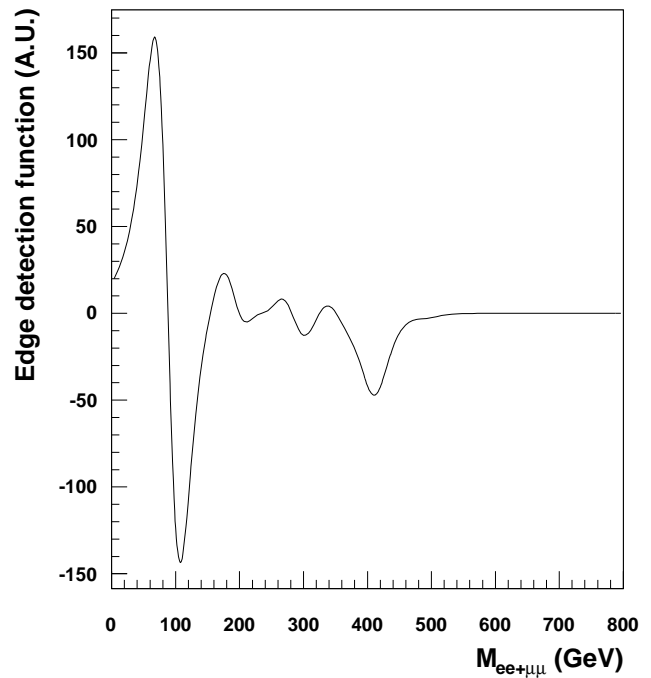

(b)

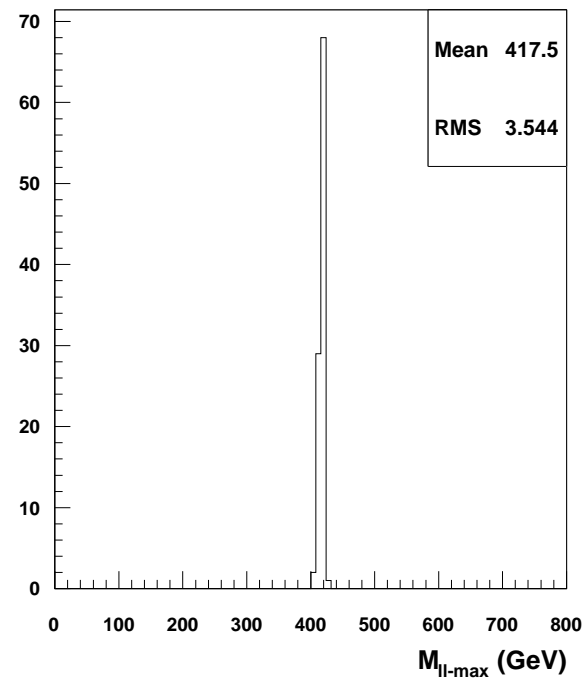

(c)

FIG. 8: (a) Histogram of events with two jets, two isolated leptons and missing energy after cuts (but including the $Z$ peak) as a function of $m_{\ell \ell}$ for the LNM-seq benchmark in a simulated experiment at the LHC with $\sqrt{s}=14$ TeV and assuming 100 $\mathrm{fb}^{-1}$ of integrated luminosity. The plot contains 726 events (502 applying the cut on the $Z$ peak). (b) Data filtering of the data in (a) through the function given in Eq.12), plotted as a function of the edge position guess and for a fixed value of the width parameter $\sigma=30 \mathrm{GeV}$. The endpoint $m_{\ell \ell}^{\max }$ of the distribution is represented by the rightmost minimum, and is reported on the data histogram in (a) with a vertical solid line. (c) Frequency histogram for the outcome of $m_{\ell \ell}^{m a x}$ for 100 random pseudo-experiments identical to the particular one plotted in (a) and (b).

a determination of $p^{\prime}$. We have verified that in practice this measurement is not possible because of the large fluctuations in the determination of the density due to the low number of points. 


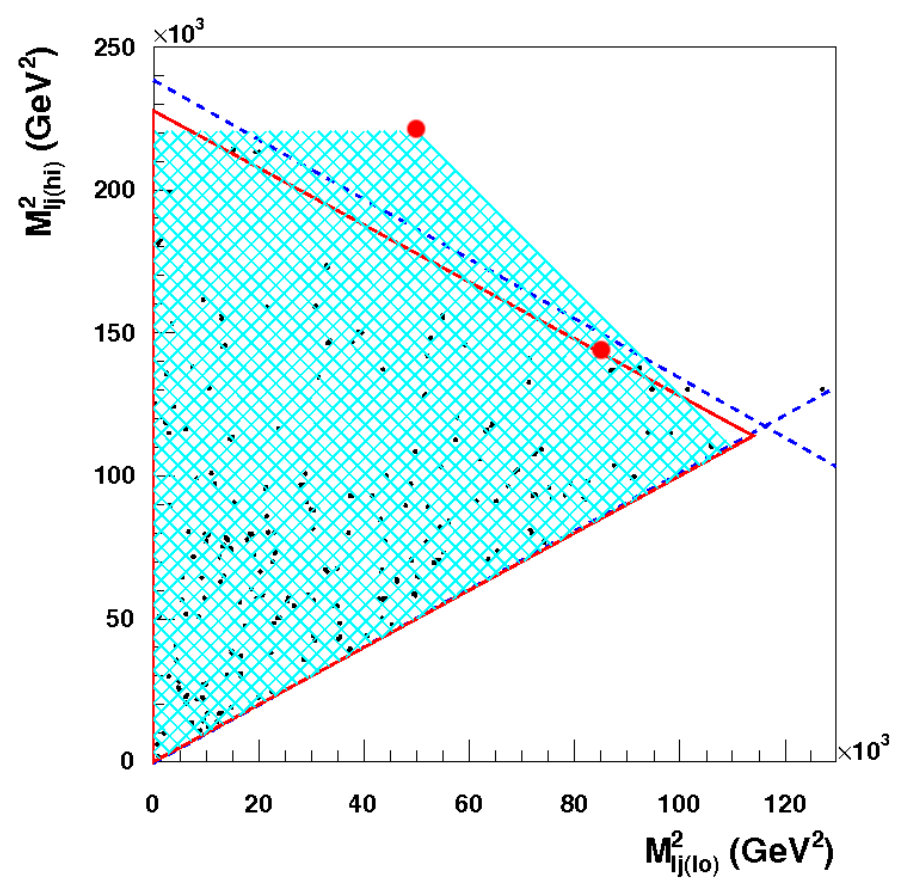

(a)
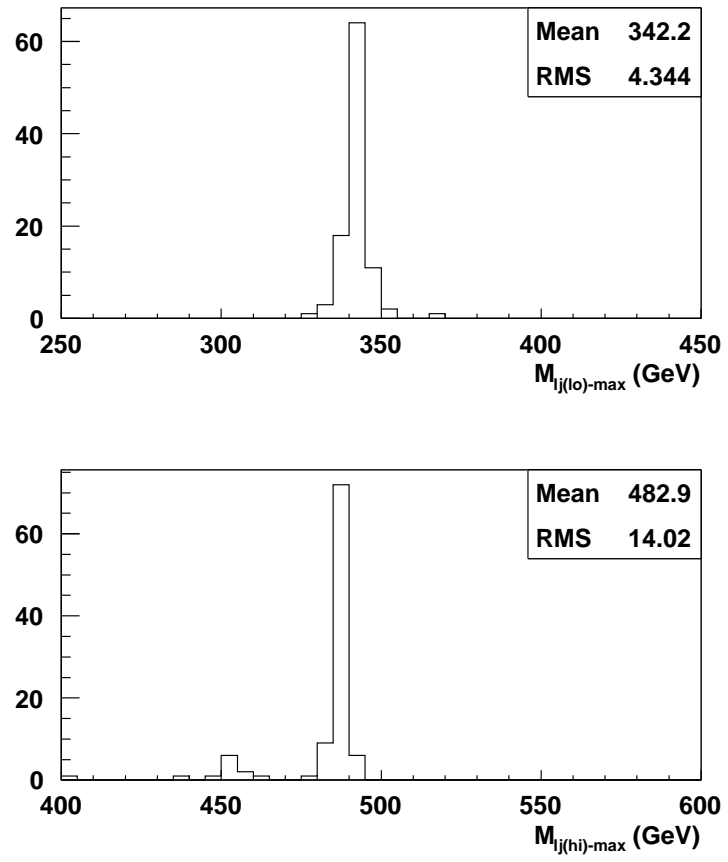

(b)

FIG. 9: (a) Two-dimensional distribution in the plane $m_{(e+\mu) j(l o)}^{2}-m_{(e+\mu) j(h i)}^{2}$ of the events plotted in Fig $8(\mathrm{a})$ that lie to the left of the value of $m_{\ell \ell}^{\max }$ determined in Fig. 8(b) and when the cut on the $Z$ peak is applied. The plot contains 497 events. The (red) solid line represents the expected boundary for LNM-seq, while the (blue) dashed lines are the fits of the boundaries, when the shape is assumed to be a triangular one (see text). The shaded area is the expected boundary for the duplicated model LNM-seq ${ }^{\prime}$. The two filled circles show the positions of the point $\left(n^{\prime}, p^{\prime}\right)$ for LNM-seq and LNM-seq'. Although for LNM-seq the exact position of $\left(n^{\prime}, p^{\prime}\right)$ cannot be measured, the fact that it lies on the boundary of the triangular shape is sufficient to break the degeneracy between the two duplicate mass patterns LNM-seq and LNM-seq' (see Table III and text). (b) Frequency histogram for the output of the quantities $m_{j \ell(l o)}^{\max }$ and $m_{j \ell(h i)}^{\max }$ for $100 \mathrm{random}$ pseudo-experiments identical to the particular one plotted in (a). In each pseudo-experiment $m_{j \ell(l o)}^{\max }$ and $m_{j \ell(h i)}^{\max }$ are obtained as the crossings of the fitted lines with the axes. 
with the LNM-seq' model is easily broken, since the expected shape for the LNM-seq benchmark, represented in Fig. 9 (a) by the (red) solid triangle, is very different from the corresponding one for LNM-seq' shown as the shaded area in the same figure. As a consequence of this the following relation among the masses:

$$
0<\frac{m_{\tilde{l}}}{m_{\chi_{i}}}<\frac{m_{\chi}}{m_{\tilde{l}}},
$$

which corresponds to the particular trapezoidal shape of the LNM-seq' benchmark, can be safely discarded.

The distribution of points in Fig 9(a) is clearly not dense enough to saturate the vertexes of the triangle ${ }^{3}$, so strictly speaking other kinematic regions different than the LNM-seq benchmark cannot be ruled out (for a summary of shapes corresponding to different kinematic situations see for instance Fig. 8 of [39]). However the shape is very compatible to a isosceles triangle as in the LNM-seq benchmark. Moreover in the LNM-seq case the possibility that the triangular shape is due to an off-shell sequential decay can be easily excluded on dynamical grounds. In fact the branching ratio of the off-shell sequential decay drops by at least two orders of magnitude compared to the on-shell situation. In this case, in order to detect a few hundreds events in the sequential channel as in Figs 9(a), 10(a) the production cross section would need to be much larger than in the LNM-seq, say in the range of a few tens pb. This in turns would lead to a dramatic enhancement of branched decays that in this case would be the dominant ones. In the LNM-seq scenario this would lead to a huge number of events showing up in the $Z$ or Higgs peaks when plotted as a function of $m_{\ell \ell}$. The non observation of such an excess would easily allow to rule out that the events plotted in Fig $9(\mathrm{a})$ are due to off-shell decays.

In such a predicament we then propose to make the educated guess that the shape is a triangle and that the sequential decay is on shell. In this case, assuming that the boundaries of the region are straight lines, the edgedetection method that we used for the $m_{\ell \ell}$ one-dimensional histogram can be modified to find the position of the edges in the two-dimensional $x-y$ plane by minimizing:

$$
\pm \sum_{i=1}^{N_{\text {data }}} f\left(y_{i}-a * x_{i}-b, \mu, \sigma\right) .
$$

The sign ' \pm ' should be chosen depending on the observed slope of the boundary.

The result of the above procedure is shown in Fig. 9(a) where the fitted straight boundaries are represented by the dashed (blue) lines. In this figure the two corresponding endpoints $m_{j \ell(l o)}^{\max }, m_{j \ell(\text { hi })}^{\max }$ are then obtained as the crossings of the boundaries with the two axes. In Fig 9(b) the same procedure is repeated for 100 pseudo-experiments identical to the one shown in Fig $9(\mathrm{a})$, and the frequency histogram for the output values of $m_{j \ell(l o)}^{\max }$ and $m_{j \ell(h i)}^{\max }$ are given. The corresponding determination for $m_{j \ell(l o)}^{\max }$ and $m_{j \ell(h i)}^{\max }$ is reported in parenthesis in Table III.

The determination of the last two endpoints $m_{j \ell \ell}^{\max }$ and $m_{j \ell \ell(\theta<\pi / 2)}^{\min }$ is finally discussed in Fig. 10, As in the previous figure the (red) solid line represents the expected boundary for the LNM-seq benchmark, while the shaded area is the corresponding one for the duplicate model LNM-seq'. Also in this case the upper and lower boundaries of the region covered by the simulated data are compatible with straight lines. This is broadly consistent with the guess that the kinematic region of Fig, $10(\mathrm{a})$ is due to an on-shell decay [39]. When the boundaries are intersected with the value of $m_{\ell \ell}^{\max }$ determined in Fig $8(\mathrm{~b})$, both $m_{j \ell \ell}^{\max }$ and $m_{j \ell \ell(\theta<\pi / 2)}^{\min }$ can be obtained. Finally, in Fig 10(b) repeating the same procedure for 100 pseudo-experiments identical to that of Fig 10(a) the frequency histograms for the output values of $m_{j \ell \ell}^{\max }, m_{j \ell \ell(\theta<\pi / 2)}^{\min }$, and of the slopes of the upper and lower boundaries is obtained. The ensuing determinations of $m_{j \ell \ell}^{\max }$ and $m_{j \ell \ell(\theta<\pi / 2)}^{\min }$ are given in parenthesis in Table III

Once the 5 endpoints $m_{\ell \ell}^{\max }, m_{j \ell(\text { hi })}^{\max }, m_{j \ell(l o w)}^{\max }, m_{j \ell \ell}^{\max }, m_{j \ell \ell(\theta<\pi / 2)}^{\min }$ are determined (notice that, as mentioned before, since the relation of Eq. (11) holds, only four of them are independent) they can be used to determine the masses. The mass inversion is obtained in a straightforward way by simulating a large number of random values of the four masses $m_{\chi}, m_{\tilde{l}}, m_{\chi_{i}}, m_{\tilde{q}}$ and plotting the histogram of the mass combinations whose theoretical values of the endpoints fall within the measured ranges. The result of such an inversion is shown in Fig. 11. As expected, this procedure

\footnotetext{
3 If it were so the endpoints would be observable in the correspondent one-dimensional projections of the distribution, without the need to resort to two-dimensions in the first place!
} 


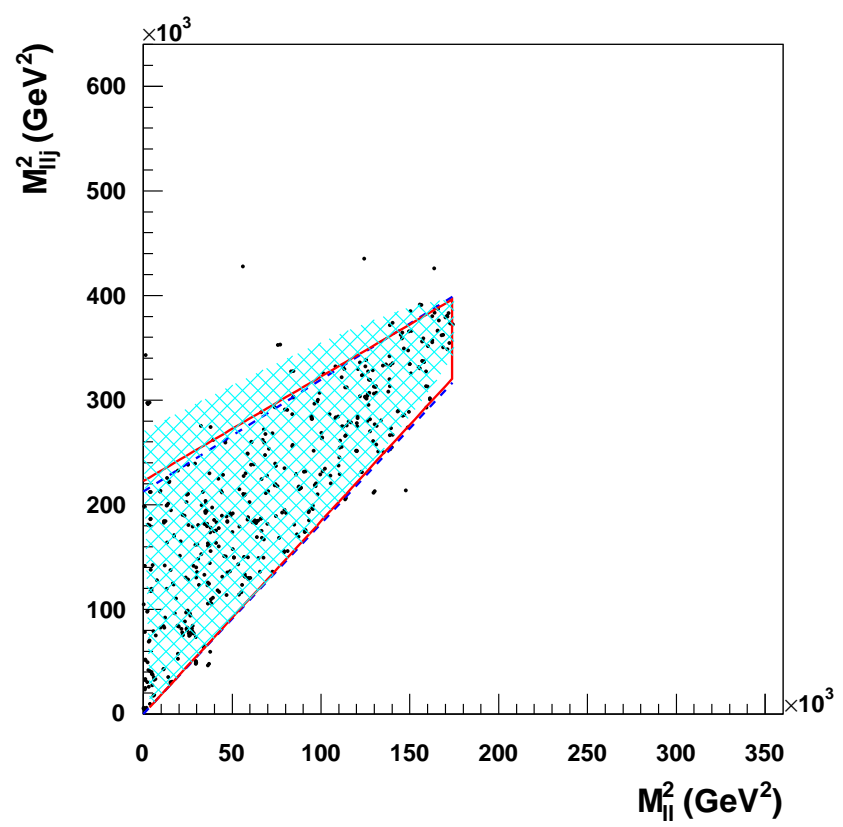

(a)
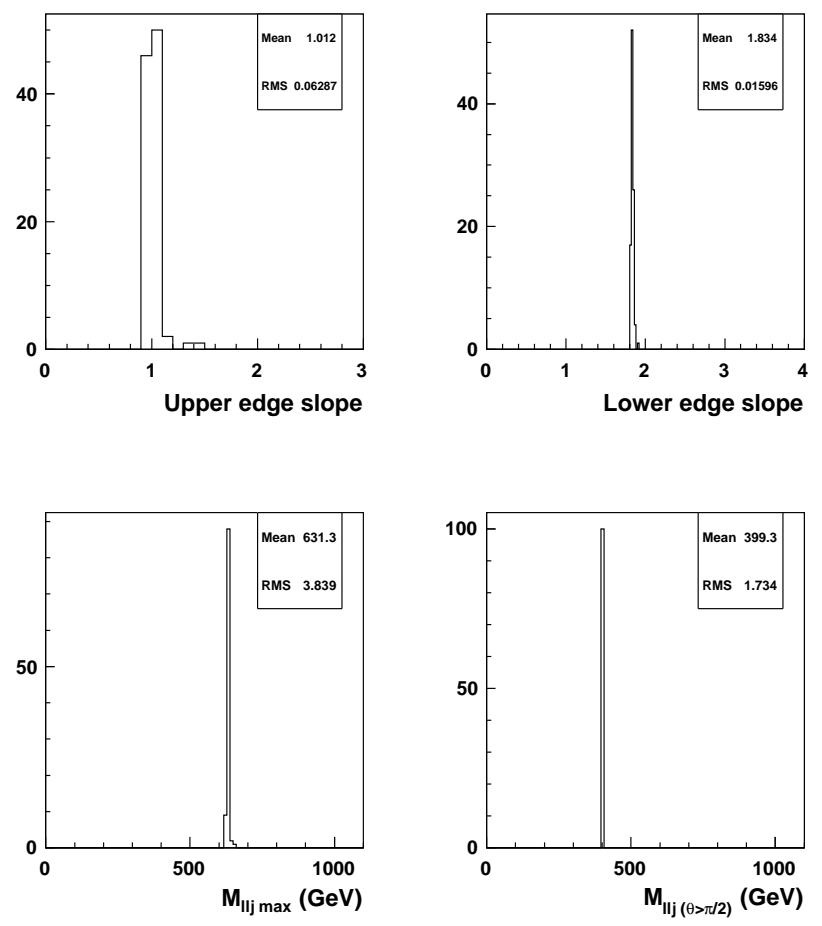

(b)

FIG. 10: (a) Two-dimensional distribution of the same events of Fig. 9(a) in the plane $m_{e e+\mu \mu}^{2}-m_{(e e+\mu \mu) j}^{2}$. The (red) solid line represents the expected boundary. The (blue) dashed lines are the fits of the upper and lower boundaries when they are assumed to be straight lines (see text). The value of $m_{\ell \ell}^{\max }$ is the one determined in Fig. 8(b) The shaded area is the boundary for the duplicated model LNM-seq'. (b) Frequency histogram for the outcome of the slopes of the upper and lower boundaries of the region in (a) and of the endpoints $m_{\ell \ell j}^{\max }$ and $m_{\ell \ell j(\theta>\pi / 2)}^{\min }$, for 100 random pseudo-experiments identical to the particular one plotted in (a). The latter quantities are obtained as the crossing points of the relevant fitted boundary lines. 

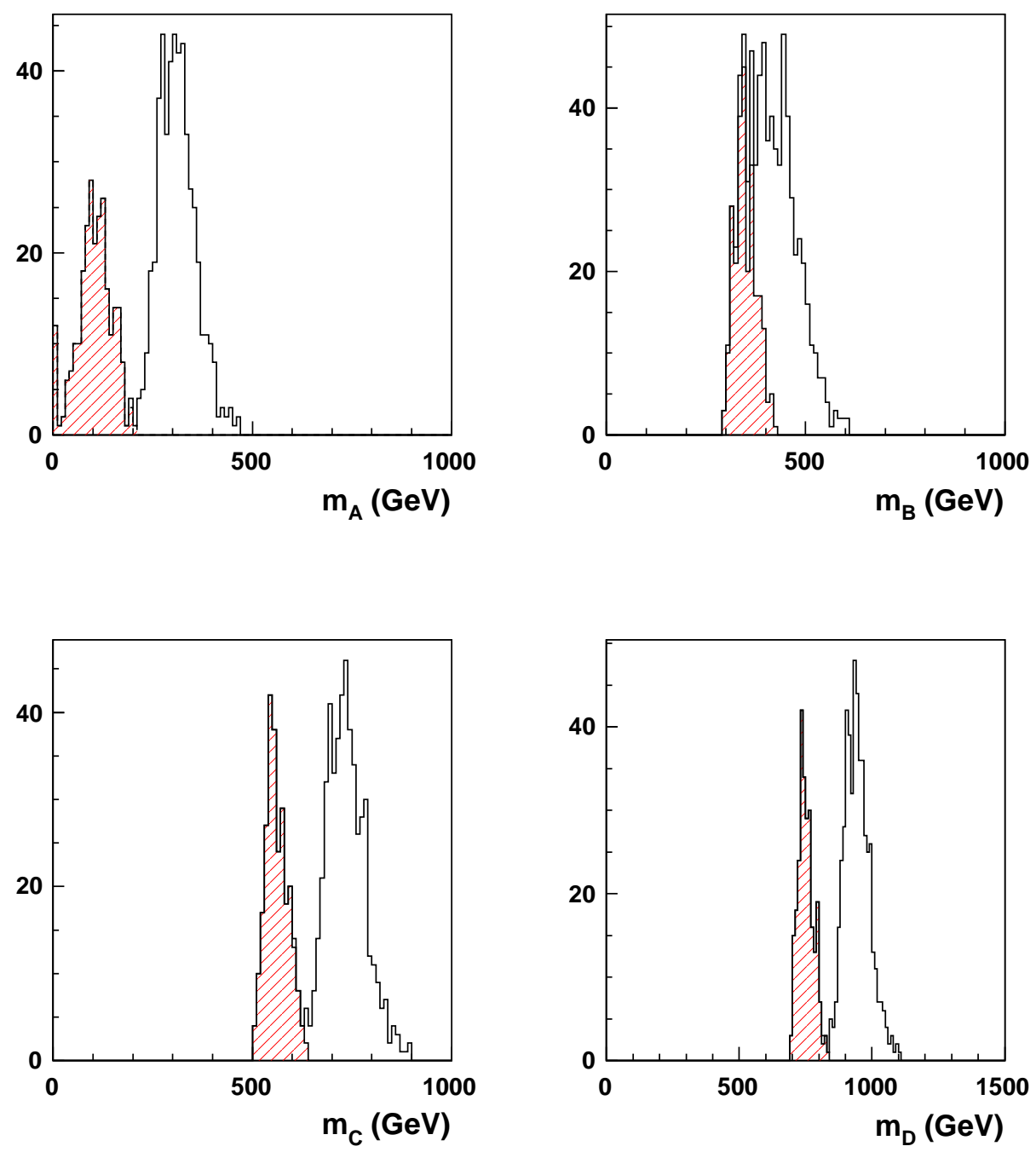

FIG. 11: Mass determination for the particles of the sequential decay obtained from the values of the endpoints measured using the simulated sample of Figs. 9(a) and 10(a) Upper left: $m_{\chi}$; upper right: $m_{\tilde{l}}$; lower left: $m_{\chi_{i}}$; lower right: $m_{\tilde{q}}$. In all plots the histogram bins are filled with a shaded box in correspondence to mass patterns verifying Eq. (13). Only the mass determinations corresponding to the shaded peaks are compatible to the data, namely to the shape of the data region of Fig $9(\mathrm{a})$ in the $m_{j \ell(h i)}-m_{j \ell(l o)}$ plane.

leads to two different solutions, corresponding to the LNM-seq benchmark and to the duplicate one LNM-seq'. Notice however that from the discussion of Fig $9(\mathrm{a})$ this degeneracy can be easily broken. In fact the distribution of the simulated data points in the $m_{j \ell(l o)}-m_{j \ell(h i)}$ plane is strongly inconsistent with the LNM-seq' solution, allowing to conclude that only mass patterns verifying Eq.(13) are compatible with the simulated data. In Fig [11 such mass patterns are marked by filling the bins with a shaded box. From this figure one can see that the correct solution can be clearly discriminated from the duplicate one for all the masses involved the decay. In this way from Fig. 11] we get the following determination of the masses:

$$
\begin{aligned}
m_{\chi} & =(103 \pm 43) \mathrm{GeV} \\
m_{\tilde{l}} & =(349 \pm 27) \mathrm{GeV} \\
m_{\chi_{i}} & =(561 \pm 28) \mathrm{GeV} \\
m_{\tilde{q}} & =(751 \pm 28) \mathrm{GeV} .
\end{aligned}
$$

The reconstructed value for the neutralino mass deviates remarkably from its input value. This is explained by 
the fact that the center-of-mass energy available in the sequential decays is set by the squark and is much larger than the neutralino mass. So neutralinos are produced in the relativistic regime, in which their kinematics is almost insensitive to the actual value of $m_{\chi}$. Moreover, the dependence of the kinematic endpoints on the neutralino mass is quadratic, so determining $m_{\chi}$ implies taking a square root, which worsens the accuracy compared to that with which the endpoints are measured. However, the reconstructed value for $m_{\chi}$, though deviated from the actual value, would entail the important indication that some neutral stable particle is being produced in the process, whose mass can be compatible to a light range. Notice that the similar yield to leptons of the three families observed in Figs. 6 and 7 allows to conclude, as discussed in Section III A that both the neutralino and the $\chi_{i}$ particle are of gaugino type. As explained in Section $\coprod$ this implies $m_{\chi} \simeq M_{1}$ and $m_{\chi_{i}} \simeq M_{2}$, so the reconstructed mass spectrum would be incompatible with neutralinos in SUGRA scenarios, since it would point toward a ratio $M_{1} / M_{2} \lesssim 0.5$, in disagreement with what expected in models where gaugino masses are unified at the GUT scale.

\section{CONCLUSIONS}

Signals of relic particles in direct DM searches raise the interest for masses of these relic particles in the range 7-8 $\mathrm{GeV} \lesssim m_{\chi} \lesssim 50 \mathrm{GeV}$. This is actually the mass range compatible with the annual-modulation effect measured by the DAMA collaboration [11], when this effect is interpreted in terms of DM particles with an elastic coherent interaction with nuclei. If the positive results of other experiments of DM direct detection are taken into account (CDMS 9], CoGeNT [8], CRESST [10]) the mass range restricts to $7-8 \mathrm{GeV} \lesssim m_{\chi} \lesssim 15-20 \mathrm{GeV}[13]$. These experimental results are fitted quite well by the Light Neutralino Model [1, 2] that is an effective Minimal Supersymmetric extension of the Standard Model at the electroweak scale without requirement of a gaugino-mass unification at a grand unification scale. At variance with Supergravity-inspired (SUGRA) models, in the LNM the neutralino mass can be as small as about $9 \mathrm{GeV}$, as discussed in the Introduction.

In view of the interest of these light neutralinos in the phenomenology related to DM direct detection, in the present paper we have addressed the problem of a search at the LHC for a neutralino of very light mass. A preliminary analysis in this direction was performed in Ref. [15]. There, specific scenarios and benchmarks within the LNM and dictated by relevant cosmological properties were considered, and the relevant expected event rates determined; however, no specific analyzes of the signal/background ratios and of kinematical distributions were performed.

In the present paper we have extended the investigation of Ref. [15] by making use of numerical simulations to estimate in a realistic way the detectability of light neutralinos at the LHC over the SM background and to show what information about the masses of SUSY particles can be extracted from the data. Within the sequential and branched decay chains that constitute the typical processes by which neutralinos can be searched for at the LHC, we have singled out the most dominant contributions in the context of the LNM. For this aspect, the analytical expressions for the light neutralino spectroscopy reported in Sect. II have been exploited.

We have then selected a benchmark (dubbed LNM-seq) with the specific feature of belonging to a value (around $10 \mathrm{GeV}$ ) of the neutralino mass that is representative of the neutralino mass values in the low-side of the $m_{\chi}$ ranges mentioned above. In terms of this benchmark we have investigated which are the expectations for having some signals at the LHC at different stages of the LHC operation.

We have found that with the integrated luminosity $\mathcal{L} \simeq 5 \mathrm{fb}^{-1}$ that would be collected at the end of the 2011 run at a center-of-mass energy of $7 \mathrm{TeV}$, the LNM-seq benchmark is expected to provide a slight excess over the background, namely at the level of $\mathrm{a} \simeq 3.2 \sigma$ significance, assuming that our estimation on the background has a $5 \%$ relative uncertainty. As discussed in Sect. $\mathrm{V}$, this would not be sufficient to draw any conclusions on the mass and properties of the neutralino.

We have then analyzed the prospects in terms of the integrated luminosity of $\mathcal{L} \simeq 100 \mathrm{fb}^{-1}$ that might be reached by the LHC at the end of its $14 \mathrm{TeV}$ run. To this purpose a detailed analysis has been performed by employing one-dimensional and two-dimensional distributions with the scope of establishing how the inputs of the LNM-seq benchmark can be reconstructed by the determinations of the relevant end-points in the various mass distributions. The problem of the disentanglement of the true solution from the duplicate solution in the inversion procedure from the end-point values to the model parameters has been addressed.

The main result concerns the reconstruction of the neutralino mass that finally turns out to be determined as $\left(m_{\chi}\right)_{\text {rec }}=103 \pm 43 \mathrm{GeV}$. This value deviates remarkably from the input value for $m_{\chi}$, but this result is not surprising in view of the difficulty in reconstructing the mass of light stable particles in relativistic events. However, the 
reconstructed value for $m_{\chi}$, though deviated from the actual value, would entail the important indication that some neutral stable particle is being produced in the process, whose mass can be compatible to a light range. Moreover, the reconstructed masses would suggest $M_{1} / M_{2} \lesssim 0.5$, in disagreement with what expected in SUGRA models where gaugino masses are unified at the GUT scale.

\section{Acknowledgments}

A.B. and N.F. acknowledge Research Grants funded jointly by Ministero dell'Istruzione, dell'Università e della Ricerca (MIUR), by Università di Torino and by Istituto Nazionale di Fisica Nucleare within the Astroparticle Physics Project (MIUR contract number: PRIN 2008NR3EBK; INFN grant code: FA51). S.S. acknowledges support by NRF with CQUEST grant 2005-0049049 and by the Sogang Research Grant 2010. N.F. acknowledges support of the spanish MICINN Consolider Ingenio 2010 Programme under grant MULTIDARK CSD2009- 00064. S.C. acknowledges support from the Korean National Research Foundation NRF-2010-0015467.

\section{Appendix A: Analytic expressions of kinematic endpoints}

We give here for completeness the analytic expressions used to calculate the kinematic endpoints in TableII] These formulae are taken from Ref. [39].

$$
\begin{aligned}
& \left(m_{\ell \ell}^{\max }\right)^{2}=m_{\tilde{q}}^{2} R_{\chi_{i} \tilde{q}}\left(1-R_{\tilde{l}_{\chi_{i}}}\right)\left(1-R_{\chi \tilde{l}}\right) \\
& \left(m_{j \ell \ell}^{\max }\right)^{2}= \begin{cases}m_{\tilde{q}}^{2}\left(1-R_{\chi_{i} \tilde{q}}\right)\left(1-R_{\chi_{\chi_{i}}}\right), & \text { for } R_{\chi_{i} \tilde{q}}<R_{\chi_{\chi_{i}}}, \\
m_{\tilde{q}}^{2}\left(1-R_{\tilde{l} \chi_{i}}\right)\left(1-R_{\chi \tilde{l}} R_{\chi_{i} \tilde{q}}\right), & \text { for } R_{\tilde{l} \chi_{i}}<R_{\chi_{\bar{l}}} R_{\chi_{i} \tilde{q}} \\
m_{\tilde{q}}^{2}\left(1-R_{\chi \tilde{l}}\right)\left(1-R_{\tilde{l} \tilde{q}}\right), & \text { for } R_{\chi \tilde{l}}<R_{\tilde{l} \tilde{q}}, \\
m_{\tilde{q}}^{2}\left(1-\sqrt{R_{\chi \tilde{q}}}\right)^{2}, & \text { otherwise. }\end{cases} \\
& \left(m_{j \ell(l o)}^{\max }\right)^{2}= \begin{cases}\left(m_{j \ell_{n}}^{\max }\right)^{2}, & \text { for }\left(2-R_{\chi \tilde{l}}\right)^{-1}<R_{\tilde{l}_{\chi_{i}}}<1, \\
\left(m_{j \ell(e q)}^{\max }\right)^{2}, & \text { for } R_{\chi \tilde{l}}<R_{\tilde{l} \chi_{i}}<\left(2-R_{\chi \tilde{l}}\right)^{-1}, \\
\left(m_{j \ell(e q)}^{\max }\right)^{2}, & \text { for } 0<R_{\tilde{l} \chi_{i}}<R_{\chi \tilde{l}},\end{cases} \\
& \left(m_{j \ell(h i)}^{\max }\right)^{2}= \begin{cases}\left(m_{j \ell_{f}}^{\max }\right)^{2}, & \text { for }\left(2-R_{\chi_{\tilde{l}}}\right)^{-1}<R_{\tilde{l}_{\chi_{i}}}<1, \\
\left(m_{j \ell_{f}}^{\max }\right)^{2}, & \text { for } R_{\chi \tilde{l}}<R_{\tilde{l}_{\chi_{i}}}<\left(2-R_{\chi \tilde{l}}\right)^{-1}, \\
\left(m_{j \ell_{n}}^{\max }\right)^{2}, & \text { for } 0<R_{\tilde{l}_{\chi_{i}}}<R_{\chi \tilde{l}},\end{cases} \\
& \left(m_{j \ell \ell\left(\theta>\frac{\pi}{2}\right)}^{\min }\right)^{2}=\frac{1}{4} m_{\tilde{q}}^{2}\left\{\left(1-R_{\chi \tilde{l}}\right)\left(1-R_{\tilde{l} \chi_{i}}\right)\left(1+R_{\chi_{i} \tilde{q}}\right)\right. \\
& \left.+2\left(1-R_{\chi_{\chi_{i}}}\right)\left(1-R_{\chi_{i} \tilde{q}}\right)-\left(1-R_{\chi_{i} \tilde{q}}\right) \sqrt{\left(1+R_{\chi \tilde{l}}\right)^{2}\left(1+R_{\tilde{l} \chi_{i}}\right)^{2}-16 R_{\chi_{\chi_{i}}}}\right\} \text {, }
\end{aligned}
$$

with: 


$$
\begin{aligned}
\left(m_{j \ell_{n}}^{\max }\right)^{2} & =m_{\tilde{q}}^{2}\left(1-R_{\chi_{i} \tilde{q}}\right)\left(1-R_{\tilde{l}_{\chi_{i}}}\right) \\
\left(m_{j \ell_{f}}^{\max }\right)^{2} & =m_{\tilde{q}}^{2}\left(1-R_{\chi_{i} \tilde{q}}\right)\left(1-R_{\chi \tilde{l}}\right) \\
\left(m_{j \ell(e q)}^{\max }\right)^{2} & =m_{\tilde{q}}^{2}\left(1-R_{\chi_{i} \tilde{q}}\right)\left(1-R_{\chi \tilde{l}}\right)\left(2-R_{\chi \tilde{l}}\right)^{-1}
\end{aligned}
$$

and $R_{l m} \equiv m_{l}^{2} / m_{m}^{2}$ with $l=\chi, \tilde{l}, \chi_{i}, \tilde{q}$.

Moreover, the quantities $\left(n^{\prime}, p^{\prime}\right)$ are given by:

$$
\begin{aligned}
& n^{\prime}=\min (n, p) \\
& p^{\prime}=\max (n, p),
\end{aligned}
$$

where:

$$
\begin{aligned}
& n=\left(m_{j \ell_{n}}^{\max }\right)^{2}=m_{\tilde{q}}^{2}\left(1-R_{\chi_{i} \tilde{q}}\right)\left(1-R_{\tilde{l}_{\chi_{i}}}\right) \\
& p=\left(m_{j \ell_{f}}^{\max }\right)^{2}=m_{\tilde{q}}^{2}\left(1-R_{\chi_{i} \tilde{q}}\right)\left(1-R_{\chi \tilde{l}}\right) .
\end{aligned}
$$

[1] A. Bottino, N. Fornengo and S. Scopel, Phys. Rev. D 67, 063519 (2003) arXiv:hep-ph/0212379; A. Bottino, F. Donato, N. Fornengo and S. Scopel, Phys. Rev. D 68, 043506 (2003) arXiv:hep-ph/0304080.

[2] N. Fornengo, S. Scopel and A. Bottino, Phys. Rev. D 83, 015001 (2011) arXiv:1011.4743 [hep-ph]].

[3] The CMS collaboration, preprint CERN-PH-EP-2011-120, arXiv:1107.5834 [hep-ph]; J. Serrano (LHCb collaboration), talk given at EPS-HEP, July 22th 2011, Grenoble.

[4] A. Bottino, F. Donato, N. Fornengo and S. Scopel, Phys. Rev. D 69, 037302 (2004) arXiv:hep-ph/0307303.

[5] R. Bernabei et al., Riv. Nuovo Cim. 26N1, 1 (2003) arXiv:astro-ph/0307403.

[6] H. K. Dreiner, S. Heinemeyer, O. Kittel, U. Langenfeld, A. M. Weber and G. Weiglein, Eur. Phys. J. C 62, 547 (2009) arXiv:0901.3485 [hep-ph]].

[7] S. Scopel, S. Choi, N. Fornengo and A. Bottino, Phys. Rev. D 83, 095016 (2011) arXiv:1102.4033 [hep-ph]].

[8] C.E. Aalseth et al. (CoGeNT Collaboration), arXiv:1106.0650 [astro-ph.CO].

[9] Z. Z. Ahmed et al. [The CDMS-II Collaboration], Science 327, 1619 (2010) arXiv:0912.3592 [astro-ph.CO]].

[10] W. Seidel, talk given at IDM10, July 2010, Montpellier, France.

[11] R. Bernabei et al., Eur. Phys. J. C 56 (2008) 333 [arXiv:0804.2741 [astro-ph]]; Eur. Phys. J. C 67, 39 (2010) arXiv:1002.1028 [astro-ph.GA]].

[12] A. Bottino, F. Donato, N. Fornengo and S. Scopel, Phys. Rev. D 81, 107302 (2010), arXiv:0912.4025[hep-ph]].

[13] P. Belli, R. Bernabei, A. Bottino, F. Cappella, R. Cerulli, N. Fornengo and S. Scopel, arXiv:1106.4667 [hep-ph], to appear in Phys. Rev. D.

[14] D. G. Cerdeno and O. Seto, JCAP 0908 (2009) 032 arXiv:0903.4677 [hep-ph]]; D. G. Cerdeno, C. Munoz and O. Seto, Phys. Rev. D 79 (2009) 023510 arXiv:0807.3029 [hep-ph]]; D. G. Cerdeno, E. Gabrielli, D. E. Lopez-Fogliani, C. Munoz and A. M. Teixeira, JCAP 0706 (2007) 008 arXiv:hep-ph/0701271; J. F. Gunion, A. V. Belikov, D. Hooper, arXiv:1009.2555 [hep-ph]]; A. V. Belikov, J. F. Gunion, D. Hooper and T. M. P. Tait, arXiv:1009.0549 [hep-ph]; C. Arina and N. Fornengo, JHEP 0711, 029 (2007) arXiv:0709.4477] [hep-ph]]; G. Belanger, S. Kraml and A. Lessa, JHEP 1107, 083 (2011) arXiv:1105.4878 [hep-ph]]; R. Foot, arXiv:1106.2688 [hep-ph]; R. Foot, Phys. Rev. D 82, 095001 (2010) arXiv:1008.0685 [hep-ph]]; Y. Mambrini, JCAP 1107 (2011) 009. arXiv:1104.4799 [hep-ph]]; Y. Mambrini, JCAP 1009 (2010) 022. arXiv:1006.3318 [hep-ph]]; E. Del Nobile, C. Kouvaris and F. Sannino, arXiv:1105.5431 [hep-ph]; S. Chang, J. Liu, A. Pierce, N. Weiner and I. Yavin, JCAP 1008 (2010) 018 arXiv:1004.0697 [hep-ph]]; J. L. Feng, J. Kumar, D. Marfatia and D. Sanford, arXiv:1102.4331v2 [hep-ph]; M. T. Frandsen, F. Kahlhoefer, J. March-Russell, C. McCabe, M. McCullough and K. Schmidt-Hoberg, arXiv:1105.3734 [hep-ph]; Y. G. Kim and S. Shin, JHEP 0905 (2009) 036 arXiv:0901.2609 [hepph]]; S. Shin, arXiv:1011.6377 [hep-ph]]; M. R. Buckley, D. Hooper and J. L. Rosner, arXiv:1106.3583 [hep-ph]; S. Andreas et al., Phys. Rev. D 82, 043522 (2010) arXiv:1003.2595 [hep-ph]]; S. Andreas, T. Hambye and M. H. G. Tytgat, JCAP 0810 (2008) 034 arXiv:0808.0255 [hep-ph]]; M.Yu. Khlopov, A.G. Mayorov and E.Yu. Soldatov, Int. J. Mod. Phys. D19, 
1385 (2010) arXiv:1003.1144]; M. S. Boucenna, S. Profumo, arXiv:1106.3368[hep-ph]]; B. Batell, M. Pospelov and A. Ritz, Phys. Rev. D 79 (2009) 115019 [arXiv:0903.3396 [hep-ph]]; C. Arina, J. Hamann and Y.Y.Y. Wong, arXiv:1105.5121 [hepph] M.T. Frandsen et al., arXiv:1105.3734 [hep-ph] S. Chang et al., JCAP 1008, 018 (2010) arXiv:1004.0697 [hep-ph]]; M. R. Buckley, D. Hooper, T. M. P. Tait, arXiv:1011.1499 [hep-ph]]; W. -Y. Keung, I. Low, G. Shaughnessy, Phys. Rev. D82 (2010) 115019. arXiv:1010.1774 [hep-ph]].

[15] A. Bottino, N. Fornengo, G. Polesello and S. Scopel, Phys. Rev. D 77, 115026 (2008) arXiv:0801.3334 [hep-ph]].

[16] A. Colaleo (ALEPH Collaboration), talk at SUSY'01, June 11-17, 2001, Dubna, Russia; J. Abdallah et al. (DELPHI Collaboration), DELPHI 2001-085 CONF 513, June 2001; LEP Higgs Working Group for Higgs boson searches, arXiv:hep-ex/0107029 LEP2 Joint SUSY Working Group, http://lepsusy.web.cern.ch/lepsusy/.

[17] A.A. Affolder et al. (CDF Collaboration), Phys. Rev. Lett. 86, 4472 (2001) arXiv:hep-ex/0010052]; V.M. Abazov et al. (D0 Collaboration), Phys. Rev. Lett. 97, 171806 (2006) arXiv:hep-ex/0608013]; ;.M. Abazov et al. (D0 Collaboration), arXiv:1106.4555 [hep-ex].

[18] S. Chatrchyan et al. [CMS Collaboration], Phys. Rev. Lett. 106, 231801 (2011) arXiv:1104.1619 [hep-ex]].

[19] J. Baglio and A. Djouadi, arXiv:1103.6247 [hep-ph].

[20] E. Barberio et al. (HFAG), arXiv:hep-ex/0603003.

[21] M. Ciuchini, G. Degrassi, P. Gambino and G.F. Giudice, Nucl. Phys. B 534, 3 (1998) arXiv:hep-ph/9806308.

[22] M. Misiak et al., Phys. Rev. Lett. 98, 022002 (2007) arXiv:hep-ph/0609232.

[23] G. W. Bennett et al. [Muon G-2 Collaboration], Phys. Rev. D 73, 072003 (2006) arXiv:hep-ex/0602035.

[24] M. Davier, A. Hoecker, B. Malaescu, C. Z. Yuan and Z. Zhang, Eur. Phys. J. C 66, 1 (2010) arXiv:0908.4300 [hep-ph]].

[25] T. Moroi, Phys.Rev. D53 (1996) 6565-6575; Erratum-ibid. D56 (1997) 4424 arXiv:hep-ph/9512396.

[26] CDF Collaboration, arXiv:1107.2304 [hep-ex].

[27] A.J. Buras, P.H. Chankowski, J. Rosiek and L. Slawianowska, Phys. Lett. B 546, 96 (2002) arXiv:hep-ph/0207241.

[28] G. Isidori and P. Paradisi, Phys. Lett. B 639, 499 (2006) arXiv:hep-ph/0605012.

[29] J.L. Rosner and S. Stone, arXiv:1002.1655 [hep-ph]]; K. Ikado et al. (Belle Collaboration), Phys. Rev. Lett. 97, 251802 (2006) arXiv:hep-ex/0604018; I. Adachi et al. (Belle Collaboration), arXiv:0809.3834 B. Aubert et al. (BaBar Collaboration), Phys. Rev. D 77, 011107R (2008) arXiv:0708.2260 [hep-ex]] and Phys. Rev. D 81, 051101 (2010) arXiv:0912.2453 [hep-ex]]

[30] B. Aubert et al. (BaBar Collaboration), Phys.Rev.D 79, 092002 (2009) [arXiv:090-2.2660 [hep-ex]].

[31] J. Dunkley et al. [WMAP Collaboration], Astrophys. J. Suppl. 180, 306 (2009) arXiv:0803.0586 [astro-ph]].

[32] D. Hooper and T. Plehn, Phys. Lett. B 562, 18 (2003) arXiv:hep-ph/0212226; G. Belanger, F. Boudjema, A. Pukhov and S. Rosier-Lees, arXiv:hep-ph/0212227

[33] ATLAS Collaboration, ATLAS detector and physics performance Technical Design Report, CERN/LHCC 99-14/15 (1999). http://atlas.web.cern.ch/Atlas/GROUPS/PHYSICS/TDR/access.html.

[34] CMS Collaboration CMS physics : Technical Design Report v.2 : Physics performance CERN-LHCC-2006-021 (2006). http://cdsweb.cern.ch/search.py?recid=942733

[35] H. Bachacou, I. Hinchliffe and F. E. Paige, Phys. Rev. D 62, 015009 (2000) hep-ph/9907518.

[36] B. C. Allanach, C. G. Lester, M. A. Parker and B. R. Webber, JHEP0009, 004 (2000) arXiv:hep-ph/0007009.

[37] G. Aad et al. [The ATLAS Collaboration], arXiv:0901.0512 [hep-ex].

[38] B. K. Gjelsten, D. J. Miller and P. Osland, JHEP 0412 (2004) 003 arXiv:hep-ph/0410303.

[39] M. Burns, K. T. Matchev and M. Park, JHEP 0905, 094 (2009) arXiv:0903.4371 [hep-ph]].

[40] V. Khachatryan et al. [CMS Collaboration], Phys. Lett. B 698, 196 (2011) arXiv:1101.1628 [hep-ex]].

[41] G. Aad et al. [Atlas Collaboration], Phys. Rev. Lett. 106, 131802 (2011) arXiv:1102.2357 [hep-ex]].

[42] J. B. G. da Costa et al. [Atlas Collaboration], Phys. Lett. B 701, 186 (2011) arXiv:1102.5290 [hep-ex]].

[43] S. Chatrchyan et al. [CMS Collaboration], arXiv:1106.0933 [hep-ex]; JHEP 1107, 113 (2011) [arXiv:1106.3272 [hep-ex]]; Preprint CERN-PH-EP-2011-065, arXiv:1106.4503 [hep-ex].

[44] G. Aad et al. [ATLAS Collaboration], Eur. Phys. J. C 71, 1682 (2011) arXiv:1103.6214 [hep-ex]]; Phys. Lett. B 701, 398 (2011) arXiv:1103.4344 [hep-ex]].

[45] W. Beenakker, R. Hopker, M. Spira and P. M. Zerwas, Nucl. Phys. B 492 (1997) 51 arXiv:hep-ph/9610490; http://www.thphys.uni-heidelberg.de/ plehn/prospino/

[46] http://www.nhn.ou.edu/ isajet/

[47] I. Hinchliffe, F. E. Paige, M. D. Shapiro, J. Soderqvist and W. Yao, Phys. Rev. D 55, 5520 (1997) arXiv:hep-ph/9610544;

[48] E. R. Davies, Machine Vision: Theory, Algorithms, Practicalities, Academic Press, 1990. 